\title{
High-resolution finite compact difference schemes for hyperbolic conservation laws
}

\author{
Yiqing Shen *, Guowei Yang, Zhi Gao \\ LHD, Institute of Mechanics, Chinese Academy of Sciences, No.15 Beisihuanxi Road, Beijing 100080, PR China
}

Received 20 April 2005; received in revised form 18 November 2005; accepted 21 November 2005

Available online 28 December 2005

\begin{abstract}
A finite compact (FC) difference scheme requiring only bi-diagonal matrix inversion is proposed by using the known high-resolution flux. Introducing TVD or ENO limiters in the numerical flux, several high-resolution FC-schemes of hyperbolic conservation law are developed, including the FC-TVD, third-order FC-ENO and fifth-order FC-ENO schemes. Boundary conditions formulated need only one unknown variable for third-order FC-ENO scheme and two unknown variables for fifth-order FC-ENO scheme. Numerical test results of the proposed FC-scheme were compared with traditional TVD, ENO and WENO schemes to demonstrate its high-order accuracy and high-resolution.
\end{abstract}

(C) 2005 Elsevier Inc. All rights reserved.

Keywords: Finite compact difference scheme; Hyperbolic conservation law; Numerical simulation

\section{Introduction}

In computational fluid dynamics, several kinds of the high-resolution numerical schemes were developed to simulate the multi-scale fluid structures. The spectral method [1] has higher accuracy but its applicability is limited to simple geometries with periodic boundary conditions. The compact finite difference scheme [2] based on a compact stencil is also of relatively high-order of accuracy, but it high-order accuracy is challenged in capturing discontinuities. Some new hybrid schemes coupling the spectral method, the compact scheme or other schemes together have proposed with the idea of total variation diminishing (TVD) [3] or essentially non-oscillatory (ENO) [4,5] for decades, such as finite spectral ENO scheme [6], hybrid compact scheme [7-11] and dispersion-controlled dissipative (DCD) schemes [12].

In order to develop a numerical scheme, the following hyperbolic conservation law is usually accepted as a model equation,

$$
\begin{aligned}
& \frac{\partial u}{\partial t}+\frac{\partial f}{\partial x}=0, \\
& u(x, 0)=u^{0}(x) .
\end{aligned}
$$

\footnotetext{
* Corresponding author.

E-mail address: yqshen@imech.ac.cn (Y.Q. Shen).
} 
Its general semi-discrete form can be written as

$$
\frac{\mathrm{d} u_{j}}{\mathrm{~d} t}=-\frac{1}{\Delta x}\left(\mathbf{A}^{-1} \mathbf{B} f\right)_{j} .
$$

Cockburn and Shu [7] developed the nonlinear stable compact schemes using the TVDM (total variation diminishing in the means) property that reads

$$
T V\left(\bar{u}^{n+1}\right) \leqslant T V\left(\bar{u}^{n}\right) \text {. }
$$

The scheme requires a symmetric matrix A and the "reconstruction" of variable $u$ from the mean variable $\bar{u}$,

$$
u_{j}=\left(\mathbf{A}^{-1} \bar{u}\right)_{j} .
$$

Ravichandran [8] improved this kind of the schemes and a wider class of the compact upwind schemes was developed without the limitation of a symmetric matrix A. These compact schemes were summarized in Ref. [8], and Tolstykh's third-order compact scheme with one point upwind numerical flux [14,17], Cockburn and Shu's third-order compact with 2-point fully upwind flux [7], Ma and Fu's third-order compact scheme with 2-point upwind weighted flux and fifth-order compact scheme with 4-point upwind weighted flux $[15,18]$ can be taken to be in the same family. In addition, Adams and Shariff [9] proposed a hybrid compact-ENO scheme for shock-turbulence interaction problems. Following the same basic approach, Pirozzoli [10] derived a conservative hybrid compact-WENO scheme. Ren [11] presented a fifth-order conservative hybrid compact-WENO scheme for shock-capturing calculation, which is constructed through the weighted average of the conservative compact scheme and WENO scheme. Jiang [12] proposed a class of the dispersion-controlled dissipative schemes based on dispersion conditions [13], and the schemes are a kind of hybrid upwind schemes, and can be used to capture shocks without any numerical oscillations and free parameters, but not compact.

The tri-diagonal matrix inversion is required in applications of the mentioned above schemes, and some hybrid schemes need a smoothness indicator to switch to schemes in shock-capturing scheme whenever necessary. In the paper, a finite compact difference method is proposed based on the special upwind compact relation requiring only bi-diagonal matrix inversion, and its flow flux can be calculated by using the adjacent known flux, and high-resolution FC-TVD, FC-ENO schemes are constructed with the TVD or ENO limiters.

\section{Numerical methodology}

Take the scalar hyperbolic conservation equation (1) as example, the flux function $f(u)$ can be split into two parts, i.e., $f(u)=f^{+}(u)+f^{-}(u)$ with $\mathrm{d} f^{+}(u) / \mathrm{d} u \geqslant 0$ and $\mathrm{d} f^{-}(u) / \mathrm{d} u \leqslant 0$. The semi-discrete conservative difference equation of (1) in a general form can be written as

$$
\frac{\mathrm{d} u_{j}}{\mathrm{~d} t}+\frac{1}{\Delta x}\left(h_{j+1 / 2}-h_{j-1 / 2}\right)=0,
$$

where the numerical flux is defined as $h_{j+1 / 2}=h_{j+1 / 2}^{+}+h_{j+1 / 2}^{-}$. One kind of the upwind compact schemes is expressed as

$$
\begin{aligned}
& \alpha^{+} F_{j-1}^{+}+\beta^{+} F_{j}^{+}=\frac{1}{\Delta x} \sum_{n=-r}^{r} a_{n}^{+} f_{j+n}^{+}, \\
& \alpha^{-} F_{j+1}^{-}+\beta^{-} F_{j}^{-}=\frac{1}{\Delta x} \sum_{n=-r}^{r} a_{n}^{-} f_{j+n}^{-},
\end{aligned}
$$

where $F_{j}^{ \pm}=\frac{\partial f_{j}^{ \pm}}{\partial x}$. With application of Taylor expansions, it is easy to show the scheme is of third-order accuracy in three grid point stencil $(r=1)$ if these coefficients are defined as

$$
\begin{aligned}
& \alpha^{+}=2, \quad \beta^{+}=4, \quad a_{-1}^{+}=-5, \quad a_{0}^{+}=4, \quad a_{1}^{+}=1, \\
& \alpha^{-}=2, \quad \beta^{-}=4, \quad a_{-1}^{-}=-1, \quad a_{0}^{-}=-4, \quad a_{1}^{-}=5 \text {. }
\end{aligned}
$$


The scheme Eq. (6) is well introduced literatures, such as in Refs. [2,16]. These coefficients for a fifth-order accuracy scheme $[15,18]$ in a five grid point stencil $(r=2)$ can be given as

$$
\begin{array}{ll}
\alpha^{+}=24, & \beta^{+}=36, \quad a_{-2}^{+}=-3, \quad a_{-1}^{+}=-44, \quad a_{0}^{+}=36, \quad a_{1}^{+}=12, \quad a_{2}^{+}=-1, \\
\alpha^{-}=24, \quad \beta^{-}=36, \quad a_{-2}^{-}=1, \quad a_{-1}^{-}=-12, \quad a_{0}^{-}=-36, \quad a_{1}^{-}=44, \quad a_{2}^{-}=3 .
\end{array}
$$

In order to examine different compact difference approximations, we assume $f=a u$ and $a>0$ in Eq. (1) with the initial condition $u(x, 0)=\mathrm{e}^{\mathrm{i} k x}$. The exact solution of Eq. (1) is $u(x, t)=\mathrm{e}^{\mathrm{i} k(x-a t)}$ and the solution of different difference schemes can be obtained as

$$
u\left(x_{j}, t\right)=\mathrm{e}^{-k_{r} \frac{a t}{\Delta x}} \mathrm{e}^{\mathrm{i} k\left(x_{j}-k_{i} \frac{a t}{k \Delta x}\right)} .
$$

Fig. 1 shows variations of $k_{i}$ and $k_{r}$ of the schemes using (7), (8) and several schemes with three-point implicit operator versus scaled wave number $\alpha(\alpha=k \Delta x)$. These schemes include the third-order upwind scheme used by Cockburn et al [7], the third-order Tolstykh upwind scheme used by Ravichandran [8] and the fifth-order upwind scheme used by Pirozzoli [10]. The resolution of the fifth-order schemes is found to be better than the third-order schemes; For the third-order schemes, the phase error of scheme (7) is between the schemes of Refs. [7,8], and appears closest to the exact solution. From the curve of $k_{r}$, the dissipation error of scheme (7) is less than other two third-order schemes if $\alpha<2.3664$, and it becomes larger than the scheme of Ref. [7] when $\alpha \geqslant 2.3664$. For fifth-order schemes, both $k_{i}$ and $k_{r}$ of scheme (8) are larger than the fifth scheme of Ref. [10].

Supposing $F_{j}^{ \pm}=\frac{1}{\Delta x}\left(\hat{h}_{j+1 / 2}^{ \pm}-\hat{h}_{j-1 / 2}^{ \pm}\right)$, Eq. (6) can be re-written in conservative form,

$$
\begin{aligned}
& \hat{h}_{j+1 / 2}^{+}=\frac{1}{\beta^{+}}\left(\sum_{n=-r+1}^{r}\left(\sum_{l=n}^{r} a_{l}^{+}\right) f_{j+n}^{+}-\alpha^{+} \hat{h}_{j-1 / 2}^{+}\right), \\
& \hat{h}_{j+1 / 2}^{-}=\frac{1}{\beta^{-}}\left(\sum_{n=-r+1}^{r}\left(\sum_{l=n}^{r} a_{l}^{-}\right) f_{j+n}^{-}-\alpha^{-} \hat{h}_{j+3 / 2}^{-}\right) .
\end{aligned}
$$

Obviously, the numerical fluxes $\hat{h}_{j+1 / 2}^{+}(j=0, \ldots, N)$ and $\hat{h}_{j+1 / 2}^{-}(j=-1, \ldots, N-1)$ can be calculated directly from Eq. (10) that requires only bi-diagonal matrix inversion in characteristic direction once the numerical fluxes $\hat{h}_{-1 / 2}^{+}$and $\hat{h}_{N+1 / 2}^{-}$are given (see boundary formula in Section 2.2). And it is also easy to use TVD or ENO limiters in each $\hat{h}_{j+1 / 2}^{+}\left(\hat{h}_{j+1 / 2}^{-}\right)$to obtain the new numerical flux being of TVD or ENO properties.

\subsection{Third-order accurate finite compact TVD scheme}

Following the notation in Ref. [7], the final numerical flux is defined as

$$
h_{j+1 / 2}^{+}=f_{j}^{+}+d f_{j+1 / 2}^{+(m)}, \quad h_{j+1 / 2}^{-}=f_{j+1}^{-}-d f_{j+1 / 2}^{-(m)},
$$
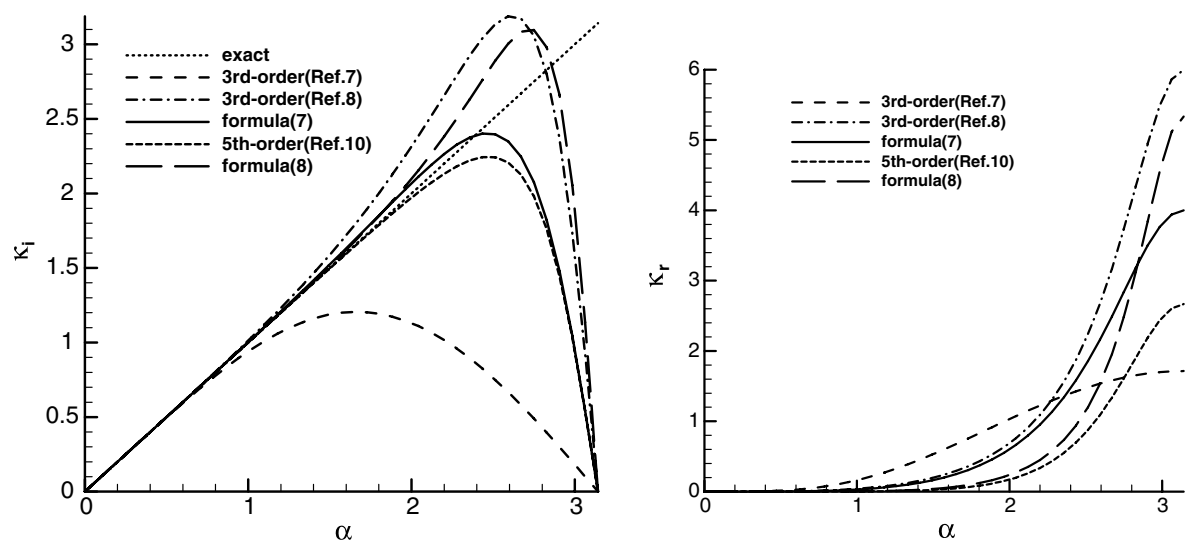

Fig. 1. Variations of of $k_{i}$ and $k_{r}$ of several schemes vs $\alpha$. 
where $d f_{j+1 / 2}^{+(m)}=m m\left(d f_{j+1 / 2}^{+}, \Delta_{+} f_{j}^{+}, \Delta_{+} f_{j-1}^{+}\right), d f_{j+1 / 2}^{-(m)}=m m\left(d f_{j+1 / 2}^{-}, \Delta_{+} f_{j}^{-}, \Delta_{+} f_{j+1}^{-}\right), d f_{j+1 / 2}^{+}=\hat{h}_{j+1 / 2}^{+}-f_{j}^{+}, d f_{j+1 / 2}^{-}=$ $f_{j+1}^{-}-\hat{h}_{j+1 / 2}^{-}, \Delta_{+} f_{j}^{ \pm}=f_{j+1}^{ \pm}-f_{j}^{ \pm}, \operatorname{mm}\left(a_{1}, a_{2}, a_{3}\right)$ is defined as

$$
m m\left(a_{1}, a_{2}, a_{3}\right)= \begin{cases}a_{1} & \text { if } \operatorname{sign}\left(a_{1}\right)=\cdots=\operatorname{sign}\left(a_{3}\right), \text { and }\left|a_{1}\right|=\min \left(\left|a_{1}\right|,\left|a_{2}\right|,\left|a_{3}\right|\right), \\ a_{2} / 2 & \text { if } \operatorname{sign}\left(a_{1}\right)=\cdots=\operatorname{sign}\left(a_{3}\right), \text { and }\left|a_{2}\right|=\min \left(\left|a_{1}\right|,\left|a_{2}\right|,\left|a_{3}\right|\right), \\ a_{3} / 2 & \text { if } \operatorname{sign}\left(a_{1}\right)=\cdots=\operatorname{sign}\left(a_{3}\right), \text { and }\left|a_{3}\right|=\min \left(\left|a_{1}\right|,\left|a_{2}\right|,\left|a_{3}\right|\right), \\ 0 & \text { otherwise. }\end{cases}
$$

The second and third values of the function $m m\left(a_{1}, a_{2}, a_{3}\right)$ will be discussed in Section 2.2. Taking $f(u)=u(x, t)$,

$$
u(x, 0)= \begin{cases}1, & -\frac{1}{5} \leqslant x \leqslant \frac{1}{5}, \\ 0, & \text { otherwise }\end{cases}
$$

as example, we can investigate the behavior of different numerical flux in the case with discontinuities. The numerical flux calculated by Eq. (10) with $N=100$ is shown in Fig. 2a and the flux oscillates in discontinuous points $x=-0.2$ and $x=0.2$. Fig. 2a also shows the flux computed with Eqs. (10) and (11), as used in Ravichandran method [8] and Ma and Fu's third order compact scheme [15]. In Fig. 2a, the region (or regions, if exist) where the first argument in Eq. 12 is chosen is only depicted. It is indicated that the region of flux is only a part $(x<-0.2)$ of the entire region. This shows that, in a large region behind the discontinuous point, the compact flux of Ravichandran method may not be used. So we use the following equation to instead of Eq. (10).

$$
\begin{aligned}
& \hat{h}_{j+1 / 2}^{+}=\frac{1}{\beta^{+}}\left(\sum_{n=-r+1}^{r}\left(\sum_{l=n}^{r} a_{l}^{+}\right) f_{j+n}^{+}-\alpha^{+} h_{j-1 / 2}^{+}\right), \\
& \hat{h}_{j+1 / 2}^{-}=\frac{1}{\beta^{-}}\left(\sum_{n=-r+1}^{r}\left(\sum_{l=n}^{r} a_{l}^{-}\right) f_{j+n}^{-}-\alpha^{-} h_{j+3 / 2}^{-}\right) .
\end{aligned}
$$

In Eq. (13), the fluxes $h_{j-1 / 2}^{+}$and $h_{j+3 / 2}^{-}$of special TVD or ENO characteristics constructed in advance may not be the compact fluxes $\hat{h}_{j-1 / 2}^{+}$and $\hat{h}_{j+3 / 2}^{-}$in Eq. (10). The obvious property of Eq. (13) is that it applies the obtained high resolution fluxes $h_{j-1 / 2}^{+}$and $h_{j+3 / 2}^{-}$to solve $\hat{h}_{j+1 / 2}^{+}$and $\hat{h}_{j+1 / 2}^{-}$, but the schemes with a three-point stencil of implicit operator cannot do so.

The flux computed with Eqs. (13) and (11) is shown in Fig. 2b. Similarly, Fig. 2b shows only the regions where the first argument in Eq. (12) is chosen. In the figure, most fluxes are displayed either before or behind

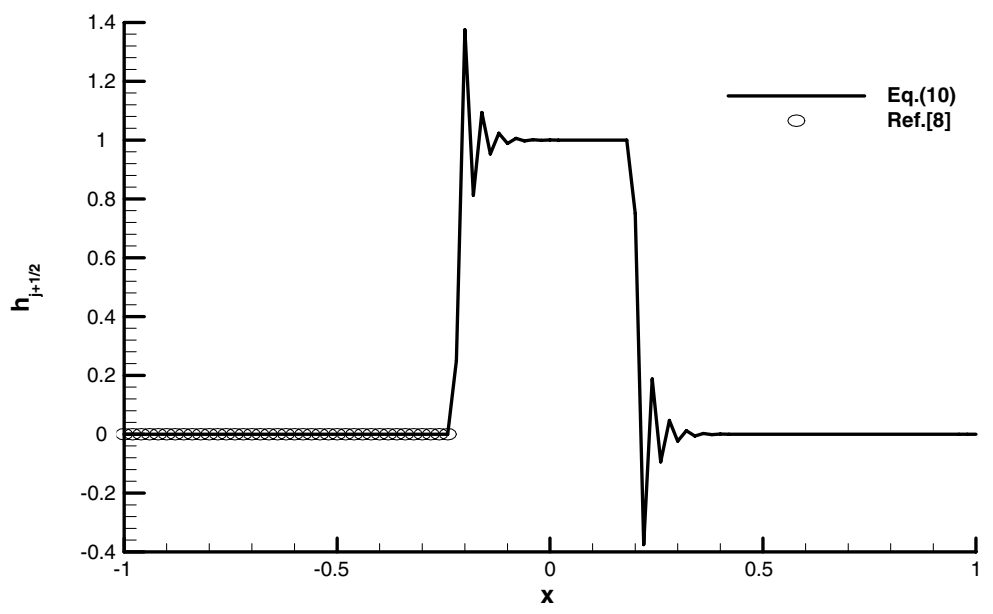

Fig. 2a. Contributions of flux function from Eq. (10) or Ref. [8] $(N=100)$. 


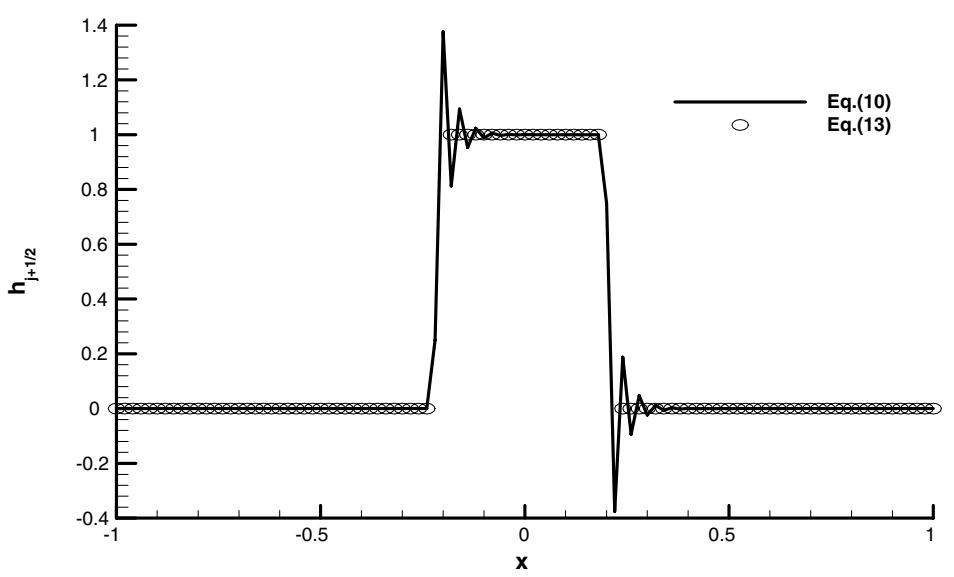

Fig. 2b. Contributions of flux function $(N=100)$.

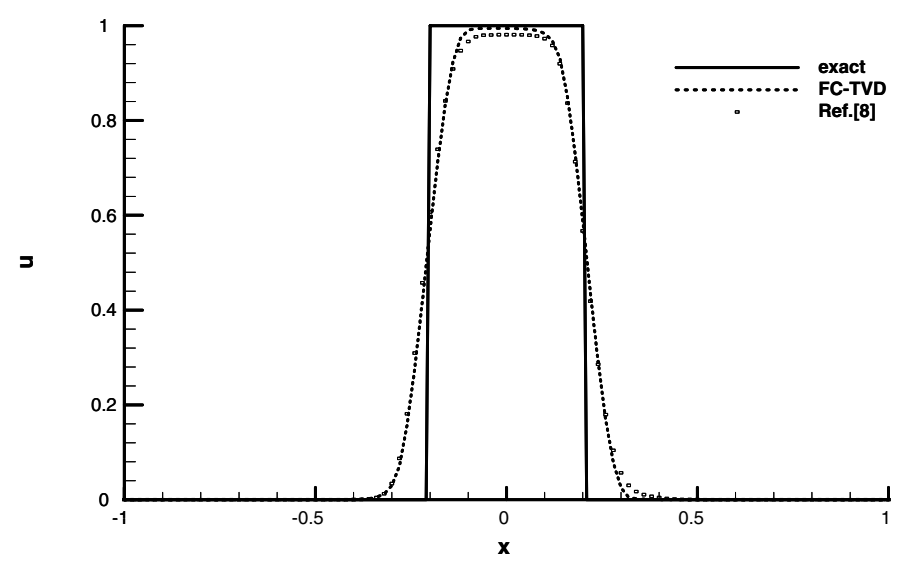

Fig. 2c. Comparisons of numerical results with an exact solution $(N=100, t=8)$.

discontinuous points. That is to say, the regions where their fluxes are calculated with Eq. (13) occupied much more part than that by Eq. (10), thus the scheme can produce better approximate accuracy.

Fig. 2c shows the comparison between numerical results of Ravichandran's scheme (Ref. [8], namely, Eqs. (13) and (11)) and FC-TVD scheme (Eqs. (10) and (11)). The FC-TVD scheme demonstrates its better performance.

Lemma 1. The semi-discrete scheme with numerical flux expressed in Eqs. (11)-(13) is TVD.

\section{Proof.}

$$
\begin{aligned}
h_{j+1 / 2}-h_{j-1 / 2} & =f_{j}^{+}+d f_{j+1 / 2}^{+(m)}+f_{j+1}^{-}-d f_{j+1 / 2}^{-(m)}-f_{j-1}^{+}-d f_{j-1 / 2}^{+(m)}-f_{j}^{-}+d f_{j-1 / 2}^{-(m)} \\
& =\Delta_{+} f_{j-1}^{+}+d f_{j+1 / 2}^{+(m)}-d f_{j-1 / 2}^{+(m)}+\Delta_{+} f_{j}^{-}-d f_{j+1 / 2}^{-(m)}+d f_{j-1 / 2}^{-(m)}=-C_{j+1 / 2} \Delta_{+} u_{j}+D_{j-1 / 2} \Delta_{+} u_{j-1},
\end{aligned}
$$

where

$$
\begin{aligned}
& C_{j+1 / 2}=-\left(\Delta_{+} f_{j}^{-}-d f_{j+1 / 2}^{-(m)}+d f_{j-1 / 2}^{-(m)}\right) / \Delta_{+} u_{j}, \\
& D_{j-1 / 2}=\left(\Delta_{+} f_{j-1}^{+}+d f_{j+1 / 2}^{+(m)}-d f_{j-1 / 2}^{+(m)}\right) / \Delta_{+} u_{j-1} .
\end{aligned}
$$

By virtue of the flux splitting, $\Delta_{+} f_{j-1}^{+} / \Delta_{+} u_{j-1} \geqslant 0$, if $\Delta_{+} u_{j-1}>0$, then $\Delta_{+} f_{j-1}^{+} \geqslant 0, d f_{j+1 / 2}^{+(m)} \geqslant 0$ and $d f_{j-1 / 2}^{+(m)} \geqslant 0$.

If $d f_{j-1 / 2}^{+(m)}=0$, then $D_{j+1 / 2}=\left(\Delta_{+} f_{j-1}^{+}+d f_{j+1 / 2}^{+(m)}\right) / \Delta_{+} u_{j-1} \geqslant 0$.

If $d f_{j-1 / 2}^{+(m)}>0$, then $d f_{j-1 / 2}^{+(m)} \leqslant \Delta_{+} f_{j-1}^{+} / 2 \leqslant \Delta_{+} f_{j-1}^{+}+d f_{j+1 / 2}^{+(m)}$. 
So that $D_{j+1 / 2}=\left(\Delta_{+} f_{j-1}^{+}+d f_{j+1 / 2}^{+(m)}-d f_{j-1 / 2}^{+(m)}\right) / \Delta_{+} u_{j-1} \geqslant 0$.

If $\Delta_{+} u_{j-1}<0$, then $\Delta_{+} f_{j-1}^{+} \leqslant 0, d f_{j+1 / 2}^{+(m)} \leqslant 0$ and $d f_{j-1 / 2}^{+(m)} \leqslant 0$.

If $d f_{j-1 / 2}^{+(m)}=0$, then $D_{j+1 / 2}=\left(\Delta_{+} f_{j-1}^{+}+d f_{j+1 / 2}^{+(m)}\right) / \Delta_{+} u_{j-1} \geqslant 0$.

If $d f_{j-1 / 2}^{+(m)}<0$, then $d f_{j-1 / 2}^{+(m)} \geqslant \Delta_{+} f_{j-1}^{+} / 2 \geqslant \Delta_{+} f_{j-1}^{+}+d f_{j+1 / 2}^{+(m)}$.

So that $D_{j+1 / 2}=\left(\Delta_{+} f_{j-1}^{+}+d f_{j+1 / 2}^{+(m)}-d f_{j-1 / 2}^{+(m)}\right) / \Delta_{+} u_{j-1} \geqslant 0$. And also, $C_{j+1 / 2} \geqslant 0$ can be deduced in the similar way. According to Harten [3], the scheme expressed in Eqs. (11)-(13) is TVD scheme, and referred as to the FC-TVD scheme in this paper.

\subsection{Third-order accurate finite compact ENO scheme}

By using the formula Eq. (23) of $h_{-1 / 2}^{+}$and $h_{N+1 / 2}^{-}$in Section 3.1 and Taylor expansion, one can easily obtain

$$
\begin{aligned}
& d f_{j+1 / 2}^{ \pm}=\frac{1}{2} \frac{\partial f_{j}^{ \pm}}{\partial x} \Delta x+\mathrm{O}\left(\Delta x^{2}\right), \\
& \Delta_{+} f_{j}^{ \pm}=\frac{\partial f_{j}^{ \pm}}{\partial x} \Delta x+\mathrm{O}\left(\Delta x^{2}\right) .
\end{aligned}
$$

In order to make the value of the limiter function $m m\left(a_{1}, a_{2}, a_{3}\right)$ has the same magnitude, the halves of the second and third arguments $\left(a_{2}\right.$ and $\left.a_{3}\right)$ in Eq. (12) are taken. Notice that the function $m m\left(a_{1}, a_{2}, a_{3}\right)$ used in Refs. $[7,8]$ is defined as

$$
m m\left(a_{1}, \ldots, a_{k}\right)= \begin{cases}s \min _{1 \leqslant i \leqslant k}\left|a_{i}\right| & \text { if } \operatorname{sign}\left(a_{1}\right)=\cdots=\operatorname{sign}\left(a_{k}\right)=s, \\ 0 & \text { otherwise. }\end{cases}
$$

Eq. (14) also shows that, in smooth regions away from critical points, $a_{2}$ and $a_{3}$ in $m m\left(a_{1}, a_{2}, a_{3}\right)$ are asymptotically of the same sign of $\alpha_{1}$ and half in its magnitude (here the critical points are defined as the points in which $f^{+}(u)_{x}=0$ or $f^{-}(u)_{x}=0$, however, the critical points in Ref. [7] are defined in means as $f^{+}(\bar{u})_{x}=0$ or $\left.f^{-}(\bar{u})_{x}=0\right)$. Therefore, for sufficiently small $\Delta x$, Eq. (11) yields

$$
h_{j+1 / 2}^{ \pm}=\hat{h}_{j+1 / 2}^{ \pm} \text {. }
$$

At critical points, the scheme is of first-order accuracy as the same to all TVD schemes. To overcome this difficulty, a modified function of $\overline{m m}\left(a_{1}, a_{2}, a_{3}\right)$, instead of $m m\left(a_{1}, a_{2}, a_{3}\right)$, is used

$$
\overline{m m}\left(a_{1}, a_{2}, a_{3}\right)= \begin{cases}a_{1} & \text { if }\left|a_{1}\right| \leqslant M \Delta x^{2}, \\ m m\left(a_{1}, a_{2}, a_{3}\right) & \text { otherwise }\end{cases}
$$

where the constant $M$ is independent of $\Delta x$. Hence the third-order finite compact ENO (FC-ENO-3) scheme is obtained.

\subsection{Fifth-order accurate finite compact ENO scheme}

The fifth-order finite compact ENO (FC-ENO-5) scheme will be constructed in this chapter. The Taylor expansions of $\hat{h}_{j+1 / 2}^{ \pm}$of Eqs. (6) and (8) read

$$
\begin{aligned}
& \hat{h}_{j+1 / 2}^{+}=f_{j}^{+}+\frac{1}{2} f_{j}^{+\prime} \Delta x+\frac{1}{12} f_{j}^{+\prime \prime} \Delta x^{2}+\mathrm{o}\left(\Delta x^{3}\right), \\
& \hat{h}_{j+1 / 2}^{-}=f_{j+1}^{-}-\frac{1}{2} f_{j+1}^{-\prime} \Delta x+\frac{1}{12} f_{j+1}^{-\prime \prime} \Delta x^{2}+\mathrm{o}\left(\Delta x^{3}\right) .
\end{aligned}
$$

So, we define a new numerical flux functions as

$$
\begin{aligned}
& h_{j+1 / 2}^{+}=f_{j}^{+}+\frac{1}{2} m\left(\Delta_{+} f_{j}^{+}, \Delta_{+} f_{j-1}^{+}\right)+D f_{j+1 / 2}^{+(m)}, \\
& h_{j+1 / 2}^{-}=f_{j+1}^{-}-\frac{1}{2} m\left(\Delta_{+} f_{j+1}^{-}, \Delta_{+} f_{j}^{-}\right)+D f_{j+1 / 2}^{-(m)},
\end{aligned}
$$


where

$$
\begin{aligned}
& D f_{j+1 / 2}^{+(m)}= \begin{cases}m m 1\left(D f_{j+1 / 2}^{+}, D_{j}^{+}, D_{j+1}^{+}\right) & \text {if }\left|\Delta_{+} f_{j}^{+}\right| \leqslant\left|\Delta_{+} f_{j-1}^{+}\right| \\
m m 2\left(D f_{j+1 / 2}^{+}, D_{j}^{+}, D_{j-1}^{+}\right) & \text {otherwise, }\end{cases} \\
& D f_{j+1 / 2}^{-(m)}= \begin{cases}m m 2\left(D f_{j+1 / 2}^{-}, D_{j+1}^{-}, D_{j+2}^{-}\right) & \text {if }\left|\Delta_{+} f_{j+1}^{-}\right| \leqslant\left|\Delta_{+} f_{j}^{-}\right|, \\
m m 1\left(D f_{j+1 / 2}^{-}, D_{j+1}^{-}, D_{j}^{-}\right) & \text {otherwise, }\end{cases} \\
& \Delta_{+} f_{j}^{ \pm}=f_{j+1}^{ \pm}-f_{j}^{ \pm}, D_{j}^{ \pm}=\Delta_{+} f_{j}^{ \pm}-\Delta_{+} f_{j-1}^{ \pm}, \\
& D f_{j+1 / 2}^{+}=\hat{h}_{j+1 / 2}^{+}-f_{j}^{+}-\frac{1}{2} m\left(\Delta_{+} f_{j}^{+}, \Delta_{+} f_{j-1}^{+}\right), \\
& D f_{j+1 / 2}^{-}=\hat{h}_{j+1 / 2}^{-}-f_{j+1}^{-}+\frac{1}{2} m\left(\Delta_{+} f_{j+1}^{-}, \Delta_{+} f_{j}^{-}\right) .
\end{aligned}
$$

And then $m\left(a_{1}, a_{2}\right)$ is defined as

$$
m\left(a_{1}, a_{2}\right)= \begin{cases}a_{1} & \text { if }\left|a_{1}\right| \leqslant\left|a_{2}\right|, \\ a_{2} & \text { otherwise }\end{cases}
$$

$m m 1\left(a_{1}, a_{2}, a_{3}\right)$ and $m m 2\left(a_{1}, a_{2}, a_{3}\right)$ are defined as

$$
\begin{aligned}
& m m 1\left(a_{1}, a_{2}, a_{3}\right)= \begin{cases}a_{1} & \text { if }\left|a_{1}\right|=\min \left(\left|a_{1}\right|,\left|a_{2}\right|,\left|a_{3}\right|\right), \\
-a_{i} / 6 & \text { otherwise and }\left|a_{i}\right|=\min \left(\left|a_{1}\right|,\left|a_{2}\right|,\left|a_{3}\right|\right),\end{cases} \\
& m m 2\left(a_{1}, a_{2}, a_{3}\right)= \begin{cases}a_{1} & \text { if }\left|a_{1}\right|=\min \left(\left|a_{1}\right|,\left|a_{2}\right|,\left|a_{3}\right|\right), \\
a_{i} / 3 & \text { otherwise and }\left|a_{i}\right|=\min \left(\left|a_{1}\right|,\left|a_{2}\right|,\left|a_{3}\right|\right) .\end{cases}
\end{aligned}
$$

Making Taylor expansion analysis of (18) yields

$$
\begin{aligned}
& D f_{j+1 / 2}^{+}= \begin{cases}-\frac{1}{6} f_{j}^{+\prime \prime} \Delta x^{2}+\mathrm{O}\left(\Delta x^{3}\right) & \text { if }\left|\Delta_{+} f_{j}^{+}\right| \leqslant\left|\Delta_{+} f_{j-1}^{+}\right|, \\
\frac{1}{3} f_{j}^{+\prime} \Delta x^{2}+\mathrm{O}\left(\Delta x^{3}\right) & \text { otherwise, }\end{cases} \\
& D f_{j+1 / 2}^{-}= \begin{cases}-\frac{1}{6} f_{j+1}^{-\prime \prime} \Delta x^{2}+\mathrm{O}\left(\Delta x^{3}\right) & \text { if }\left|\Delta_{+} f_{j+1}^{-}\right| \leqslant\left|\Delta_{+} f_{j}^{-}\right|, \\
\frac{1}{3} f_{j+1}^{-\prime \prime} \Delta x^{2}+\mathrm{O}\left(\Delta x^{3}\right) & \text { otherwise, }\end{cases} \\
& D_{k}^{+}=f_{j}^{+\prime \prime} \Delta x^{2}+\mathrm{O}\left(\Delta x^{3}\right), \quad k=j-1, j, j+1, \\
& D_{k}^{-}=f_{j+1}^{-\prime \prime} \Delta x^{2}+\mathrm{O}\left(\Delta x^{3}\right), \quad k=j, j+1, j+2 .
\end{aligned}
$$

So, in smooth regions away from the point of $f_{j}^{ \pm \prime \prime}=0$ for sufficiently small $\Delta x$, the first arguments of $m m 1\left(a_{1}, a_{2}, a_{3}\right)$ and $m m 2\left(a_{1}, a_{2}, a_{3}\right)$ will be chosen, which yields

$$
h_{j+1 / 2}^{ \pm}=\hat{h}_{j+1 / 2}^{ \pm} \text {. }
$$

Hence, the scheme is of fifth-order accuracy. Meanwhile, the coefficients of the second and third arguments of $m m 1\left(a_{1}, a_{2}, a_{3}\right)$ and $m m 2\left(a_{1}, a_{2}, a_{3}\right)$ are determined with the second-order derivative in formula (20).

To overcome the drawback of accuracy degeneration at the points of $f_{j}^{ \pm \prime \prime}=0$, we modify $\operatorname{mm} 1\left(a_{1}, a_{2}, a_{3}\right)$ and $m m 2\left(a_{1}, a_{2}, a_{3}\right)$ as

$$
\begin{aligned}
& \overline{m m 1}\left(a_{1}, a_{2}, a_{3}\right)= \begin{cases}a_{1} & \text { if }\left|a_{1}\right| \leqslant M \Delta x^{3}, \\
m m 1\left(a_{1}, a_{2}, a_{3}\right) & \text { otherwise, }\end{cases} \\
& \overline{m m 2}\left(a_{1}, a_{2}, a_{3}\right)= \begin{cases}a_{1} & \text { if }\left|a_{1}\right| \leqslant M \Delta x^{3}, \\
m m 2\left(a_{1}, a_{2}, a_{3}\right) & \text { otherwise, }\end{cases}
\end{aligned}
$$

where $M$ is a constant independent of $\Delta x$ and is set to be one in this paper. 


\section{Boundary formulations and time discretization}

\subsection{Three points boundary formulation}

Using the four points stencil $\left(x_{j-1}, x_{j}, x_{j+1}, x_{j+2}\right)$ to approximate $f_{j}^{\prime}$, we can get

$$
f_{j}^{+\prime}=\frac{-11 f_{j-1}^{+}+18 f_{j}^{+}-9 f_{j+1}^{+}+2 f_{j+2}^{+}}{6 \Delta x}+\mathrm{O}\left(\Delta x^{3}\right)
$$

then $h_{-1 / 2}^{+}$can be obtained as

$$
h_{-1 / 2}^{+}=\frac{1}{3} f_{-1}^{+}+\frac{5}{6} f_{0}^{+}-\frac{1}{6} f_{1}^{+} .
$$

Similarly, the flux

$$
h_{N+1 / 2}^{-}=-\frac{1}{6} f_{N-1}^{-}+\frac{5}{6} f_{N}^{-}+\frac{1}{3} f_{N+1}^{-} .
$$

The boundary fluxes $h_{-1 / 2}^{+}$and $h_{N+1 / 2}^{-}$require only two boundary values at the node of $x_{0}, x_{N}\left(f_{-1}^{+}\right.$for $h_{-1 / 2}^{+}$, $f_{N+1}^{-}$for $\left.h_{N+1 / 2}^{-}\right)$.

\subsection{Five points boundary formulation}

Using the six points stencil $\left(x_{j-2}, x_{j-1}, x_{j}, x_{j+1}, x_{j+2}, x_{j+3}\right)$ to approximate $f_{j}^{+\prime}$, we have

$$
f_{j}^{+\prime}=\frac{-12 f_{j-2}^{+}-65 f_{j-1}^{+}+120 f_{j}^{+}-60 f_{j+1}^{+}+20 f_{j+2}^{+}-3 f_{j+3}^{+}}{60 \Delta x}+\mathrm{O}\left(\Delta x^{5}\right) .
$$

The flux $h_{-1 / 2}^{+}$is obtained as

$$
h_{-1 / 2}^{+}=\frac{1}{60}\left(-3 f_{-2}^{+}+27 f_{-1}^{+}+47 f_{0}^{+}-13 f_{1}^{+}+2 f_{2}^{+}\right) .
$$

Similarly,

$$
h_{N+1 / 2}^{-}=\frac{1}{60}\left(2 f_{N-2}^{-}-13 f_{N-1}^{-}+47 f_{N}^{-}+27 f_{N+1}^{-}-3 f_{N+2}^{-}\right) .
$$

The boundary fluxes $h_{-1 / 2}^{+}$and $h_{N+1 / 2}^{-}$require only two boundary values $\left(f_{-2}^{+}\right.$and $f_{-1}^{+}$for $h_{-1 / 2}^{+}, f_{N+1}^{-}$and $f_{N+2}^{-}$for $\left.h_{N+1 / 2}^{-}\right)$.

In the following computations, the boundary formulation (23) is applied for the FC-TVD and FC-ENO-3 schemes, and the boundary formulation (24) is accepted in the FC-ENO-5 scheme.

\subsection{Stability analysis}

In order to determine the stability of the compact scheme (7) with boundary formulation (23) and the compact scheme (8) with boundary formulation Eq. (24), the semi-discrete approximation of Eq. (1) under the condition of the positive flux $(f=a u$ with $a>0)$ can be expressed in the compact matrix notation of Pirozzoli $[10]$ as

$$
\mathbf{u}_{t}=-\frac{a}{\Delta x} \mathbf{C A}^{-1}(\mathbf{B u}+\mathbf{b})
$$

where $\mathbf{u}=\left(u_{0}, u_{1}, \ldots, u_{N}\right)^{\mathrm{T}}$. For the scheme (7) with boundary formulation (23), 


$$
\mathbf{A}=\left[\begin{array}{llll}
1 & & & \\
2 & 4 & & \\
& \ddots & \ddots & \\
& & 2 & 4
\end{array}\right]_{N 1 \times N 1}, \quad \mathbf{B}=\left[\begin{array}{cccc}
\frac{5}{6} & -\frac{1}{6} & & \\
5 & 1 & & \\
& \ddots & \ddots & \\
& 5 & 1 & \\
& & & 5
\end{array}\right]_{N 1 \times N} \quad \mathbf{C}=\left[\begin{array}{cccc}
-1 & 1 & & \\
& \ddots & \ddots & \\
& & -1 & 1
\end{array}\right]_{N \times N 1},
$$

and $\mathbf{b}=\left(1 / 3 u_{0}, 0, \ldots, 0, u_{N+1}\right)^{\mathrm{T}}$. For the scheme (8) with boundary formulation (24),

$$
\mathbf{A}=\left[\begin{array}{ccc}
1 & & \\
24 & 36 & \\
& \ddots & \ddots \\
& 24 & 36
\end{array}\right]_{N 1 \times N 1}, \quad \mathbf{B}=\left[\begin{array}{ccccc}
\frac{47}{60} & -\frac{13}{60} & \frac{2}{60} & & \\
47 & 11 & -1 & & \\
3 & 47 & 11 & -1 & \\
\ddots & \ddots & \ddots & \ddots & \\
& 3 & 47 & 11 & -1 \\
& & 3 & 47 & 11 \\
& & & 3 & 47
\end{array}\right]_{N 1 \times N} \quad, \quad \mathbf{C}=\left[\begin{array}{ccc}
-1 & 1 & \\
& \ddots & \ddots \\
-1 & 1
\end{array}\right]_{N \times N 1},
$$

and $\mathbf{b}=\left(27 / 60 u_{-1}-3 / 60 u_{-2}, 3 u_{-1}, 0, \ldots, 0,-u_{N+1}, 11 u_{N+1}-u_{N+2}\right)^{\mathrm{T}}$, where $N 1=N+1$.

As discussed in Refs. [2,10], the stability condition for the semi-discrete equation requires that all eigenvalues of the matrix $\mathbf{S}=-\mathbf{C A}^{-1} \mathbf{B}$ have a negative real part. Figs. 3a and $\mathrm{b}$ showing numerical results of the eigenvalue spectra of matrix $S$ demonstrated that the third-order upwind scheme (7) with boundary formulation (23) and the fifth-order upwind scheme (8) with boundary equation (24) are linearly stable.

\subsection{Time discretization}

Discretizations of time-derivative are performed by both the third-order TVD Runge-Kutta method [20] for TVD, FC-TVD and FC-ENO-3 schemes, and the fourth-order Runge-Kutta for ENO, FC-ENO-5 and 5-WENO schemes.

For the ordinary differential equation

$$
\frac{\mathrm{d} u}{\mathrm{~d} t}=L(u)
$$

the third-order TVD Runge-Kutta method [20] is expressed as
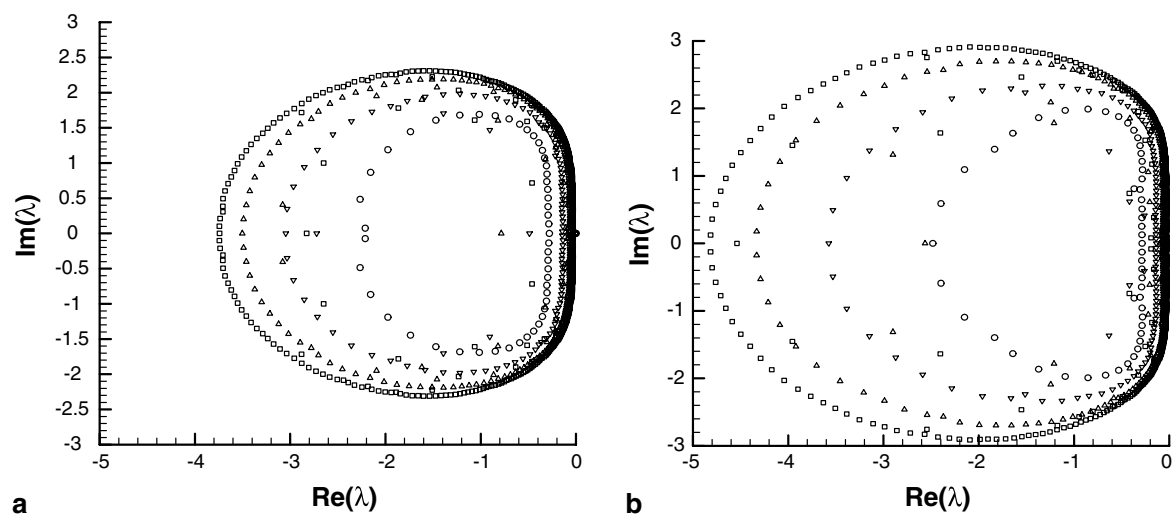

Fig. 3. Eigenvalue spectra of the upwind compact schemes, $\bigcirc: N=50 ; \nabla: N=100 ; \triangle: N=200 ; \square: N=400$, (a) The third-order upwind scheme (7) with boundary formulation (23). (b) The fifth-order upwind scheme (8) with boundary formulation (24). 


$$
\begin{aligned}
& u^{(1)}=u^{n}+\Delta t L\left(u^{n}\right), \\
& u^{(2)}=\frac{3}{4} u^{n}+\frac{1}{4} u^{(1)}+\frac{1}{4} \Delta t L\left(u^{(1)}\right), \\
& u^{n+1}=\frac{1}{3} u^{n}+\frac{2}{3} u^{(2)}+\frac{2}{3} \Delta t L\left(u^{(2)}\right) .
\end{aligned}
$$

The fourth-order Runge-Kutta method is written as

$$
\begin{aligned}
& u^{(1)}=u^{n}+\frac{1}{2} \Delta t L\left(u^{n}\right), \\
& u^{(2)}=u^{n}+\frac{1}{2} \Delta t L\left(u^{(1)}\right), \\
& u^{(3)}=u^{n}+\frac{1}{2} \Delta t L\left(u^{(2)}\right), \\
& u^{n+1}=\frac{1}{3}\left(-u^{n}+u^{(1)}+2 u^{(2)}+u^{(3)}\right)+\frac{1}{6} \Delta t L\left(u^{(3)}\right) .
\end{aligned}
$$

\section{Numerical examples}

(1) Linear transport equation. The transport equation is given as

$\frac{\partial u}{\partial t}+\frac{\partial u}{\partial x}=0, \quad-\pi \leqslant x \leqslant \pi$

$u(x, 0)=\sin x, \quad-\pi \leqslant x \leqslant \pi$, periodic boundary condition.

The example is used to examine the accuracy of FC-TVD, FC-ENO-3 and FC-ENO-5 schemes. The TVD, ENO-3 (third-order ENO) and 5-WENO (fifth-order WENO) schemes are also appplied. Accuracy comparisons are shown in Tables 1 and 2. The tables indicate that FC-TVD is more accurate than TVD, FC-ENO-3 gives third-order of accuracy and is better than FC-TVD, and FC-ENO-5 reaches the fifth-order accuracy, although its order is slightly less than the 5-WENO's, its errors decrease about 5-10

\begin{tabular}{|c|c|c|c|c|c|}
\hline Scheme & $N$ & $L_{\infty}$ error & $L_{\infty}$ order & $L_{1}$ error & $L_{1}$ order \\
\hline \multirow[t]{5}{*}{ FC-ENO-3 } & 20 & $0.2618 \mathrm{e}-2$ & - & $0.7115 e-3$ & - \\
\hline & 40 & $0.4793 e-3$ & 2.449 & $0.1021 \mathrm{e}-3$ & 2.801 \\
\hline & 80 & $0.7645 \mathrm{e}-4$ & 2.648 & $0.1334 \mathrm{e}-4$ & 2.936 \\
\hline & 160 & $0.1101 \mathrm{e}-4$ & 2.796 & $0.1687 \mathrm{e}-5$ & 2.983 \\
\hline & 320 & $0.1525 \mathrm{e}-5$ & 2.852 & $0.2143 e-6$ & 2.977 \\
\hline \multirow[t]{5}{*}{ FC-TVD } & 20 & $0.5418 \mathrm{e}-1$ & - & $0.2171 \mathrm{e}-1$ & - \\
\hline & 40 & $0.2265 \mathrm{e}-1$ & 1.258 & $0.5573 \mathrm{e}-2$ & 1.962 \\
\hline & 80 & $0.8280 \mathrm{e}-2$ & 1.452 & $0.1211 \mathrm{e}-2$ & 2.202 \\
\hline & 160 & $0.3134 \mathrm{e}-2$ & 1.402 & $0.2522 \mathrm{e}-3$ & 2.264 \\
\hline & 320 & $0.1101 \mathrm{e}-2$ & 1.509 & $0.5112 \mathrm{e}-4$ & 2.303 \\
\hline \multirow[t]{5}{*}{ TVD } & 20 & $0.6460 \mathrm{e}-1$ & - & $0.2610 \mathrm{e}-1$ & - \\
\hline & 0 & $0.2983 e-1$ & 1.115 & $0.8240 \mathrm{e}-2$ & 1.663 \\
\hline & 80 & $0.1217 \mathrm{e}-1$ & 1.293 & $0.2136 \mathrm{e}-2$ & 1.948 \\
\hline & 160 & $0.5052 \mathrm{e}-2$ & 1.268 & $0.6277 \mathrm{e}-3$ & 1.767 \\
\hline & 320 & $0.2059 \mathrm{e}-2$ & 1.295 & $0.1679 \mathrm{e}-3$ & 1.902 \\
\hline
\end{tabular}
times lower than the 5-WENO's.

(2) $\frac{\partial u}{\partial t}+\frac{\partial u}{\partial x}=0,-1 \leqslant x \leqslant 1$.

This equation is taken as the second test case there

$$
u(x, 0)=\left\{\begin{array}{ll}
1, & -\frac{1}{5} \leqslant x \leqslant \frac{1}{5}, \\
0, & \text { otherwise }
\end{array}\right. \text { with a periodic boundary condition. }
$$

Table 1

Comparisons of several schemes, $t=1$ 
Table 2

Comparisons of several schemes, $t=1$

\begin{tabular}{|c|c|c|c|c|c|}
\hline Scheme & $N$ & $L_{\infty}$ error & $L_{\infty}$ order & $L_{1}$ error & $L_{1}$ order \\
\hline \multirow[t]{6}{*}{ FC-ENO-5 } & 10 & $0.1016 \mathrm{e}-2$ & - & $0.2626 \mathrm{e}-3$ & - \\
\hline & 20 & $0.4954 \mathrm{e}-4$ & 4.358 & $0.1049 \mathrm{e}-4$ & 4.646 \\
\hline & 40 & $0.2166 e-5$ & 4.515 & $0.3693 e-6$ & 4.828 \\
\hline & 80 & $0.8497 \mathrm{e}-7$ & 4.672 & $0.1213 e-7$ & 4.928 \\
\hline & 160 & $0.2976 \mathrm{e}-8$ & 4.836 & $0.3862 \mathrm{e}-9$ & 4.973 \\
\hline & 320 & $0.9998 \mathrm{e}-10$ & 4.896 & $0.1219 \mathrm{e}-10$ & 4.986 \\
\hline \multirow[t]{6}{*}{ 5-WENO } & 10 & $0.6756 \mathrm{e}-2$ & - & $0.5061 \mathrm{e}-2$ & - \\
\hline & 20 & $0.3081 \mathrm{e}-3$ & 4.455 & $0.1577 \mathrm{e}-3$ & 5.004 \\
\hline & 40 & $0.9525 \mathrm{e}-5$ & 5.016 & $0.4511 e-5$ & 5.128 \\
\hline & 80 & $0.2970 \mathrm{e}-6$ & 5.003 & $0.1314 \mathrm{e}-6$ & 5.101 \\
\hline & 160 & $0.8681 \mathrm{e}-8$ & 5.096 & $0.3991 \mathrm{e}-8$ & 5.041 \\
\hline & 320 & $0.2297 \mathrm{e}-9$ & 5.240 & $0.1240 \mathrm{e}-9$ & 5.008 \\
\hline \multirow[t]{6}{*}{ ENO-3 } & 10 & $0.2174 \mathrm{e}-1$ & - & $0.1290 \mathrm{e}-1$ & - \\
\hline & 20 & $0.2778 \mathrm{e}-2$ & 2.968 & $0.1664 \mathrm{e}-2$ & 2.955 \\
\hline & 40 & $0.3802 \mathrm{e}-3$ & 2.869 & $0.2079 \mathrm{e}-3$ & 3.001 \\
\hline & 80 & $0.4581 \mathrm{e}-4$ & 3.053 & $0.2594 \mathrm{e}-4$ & 3.003 \\
\hline & 160 & $0.5436 e-5$ & 3.075 & $0.3225 e-5$ & 3.008 \\
\hline & 320 & $0.6897 e-6$ & 2.979 & $0.4027 \mathrm{e}-6$ & 3.002 \\
\hline
\end{tabular}

The results are shown in Figs. 4a and 4b. FC-ENO-3 shows a significant improvement in shock capturing and behaves better than TVD, and even better than ENO-3. The result of FC-ENO-5 is also slightly better than 5-WENO. The results obtained with FC-TVD and FC-ENO-3, FC-TVD schemes are not depicted in the figure because of these are almost the same.

(3) $\frac{\partial u}{\partial t}+\frac{\partial u}{\partial x}=0,-1 \leqslant x \leqslant 1$.

This equation is accepted as the third case where

$$
u(x, 0)= \begin{cases}\frac{1}{6}(G(x, \beta, z-\delta)+G(x, \beta, z+\delta)+4 G(x, \beta, z)), & -0.8 \leqslant x \leqslant-0.6, \\ 1, & -0.4 \leqslant x \leqslant-0.2, \\ 1-|10(x-0.1)|, & 0 \leqslant x \leqslant 0.2 \\ \frac{1}{6}(F(x, \alpha, a-\delta)+F(x, \alpha, a+\delta)+4 F(x, \alpha, a)), & 0.4 \leqslant x \leqslant 0.6, \\ 0, & \text { otherwise }\end{cases}
$$

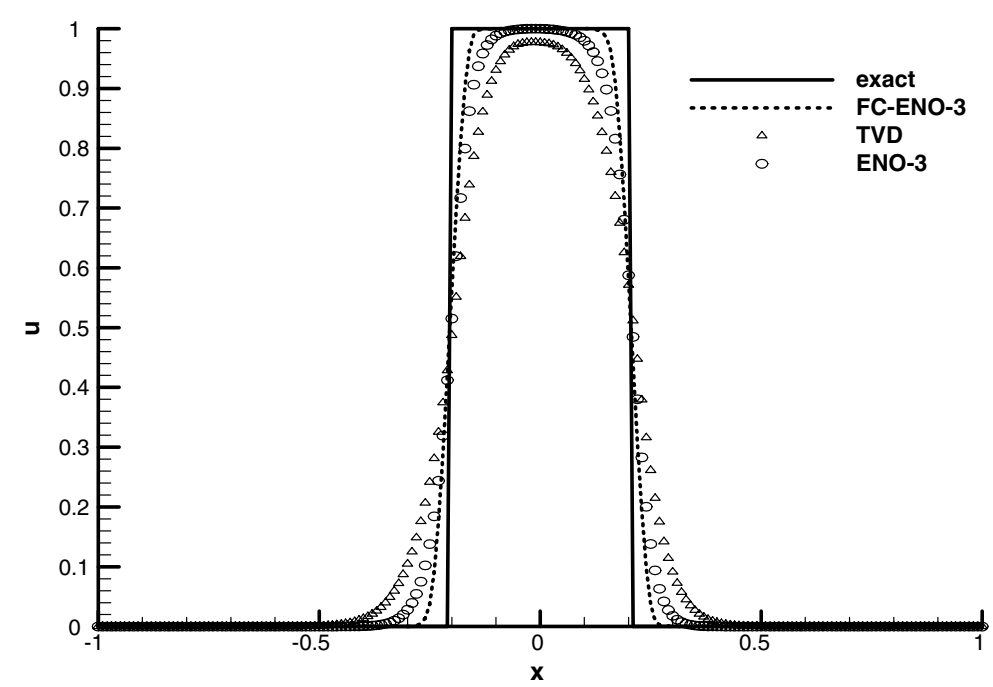

Fig. 4a. Numerical results of example (2), $N=200, t=6$. 


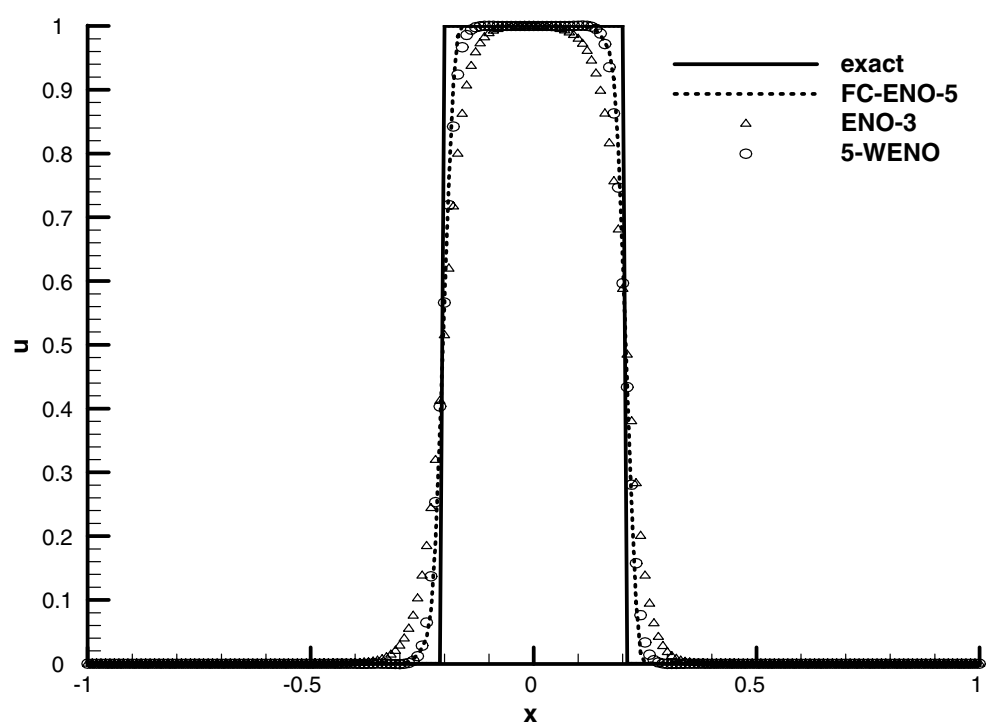

Fig. 4b. Numerical results of case $2, N=200, t=6$.

$G(x, \beta, z)=\mathrm{e}^{-\beta(x-z)^{2}}, \quad F(x, \alpha, a)=\sqrt{\max \left(1-\alpha^{2}(x-a)^{2}, 0\right)}, \quad a=0.5, z=-0.7, \delta=0.005, \alpha=10$, $\beta=\log 2 / 36 \delta^{2}$.

The solution includes a smooth but narrow combination of Gaussians, a square wave, a sharp triangle wave, and a half ellipse [19]. 200 grid points are used and the solution is intergraded up to $t=4$ in calculations. The results in Fig. 5 indicate that FC-ENO-3 performs better than TVD for four types of waves, and although the ENO-3 needs more grid points than FC-ENO-3, its numerical results are still less than FC-ENO-3 in most of regions, as shown in Fig. 5a. Fig. 5b shows that FC-ENO-3 is much better than Ravichandran's scheme. From Fig. 5c and its local enlarge figure in Fig. 5d, the FC-ENO-5 gives the most accurate results in all schemes for four types of waves.

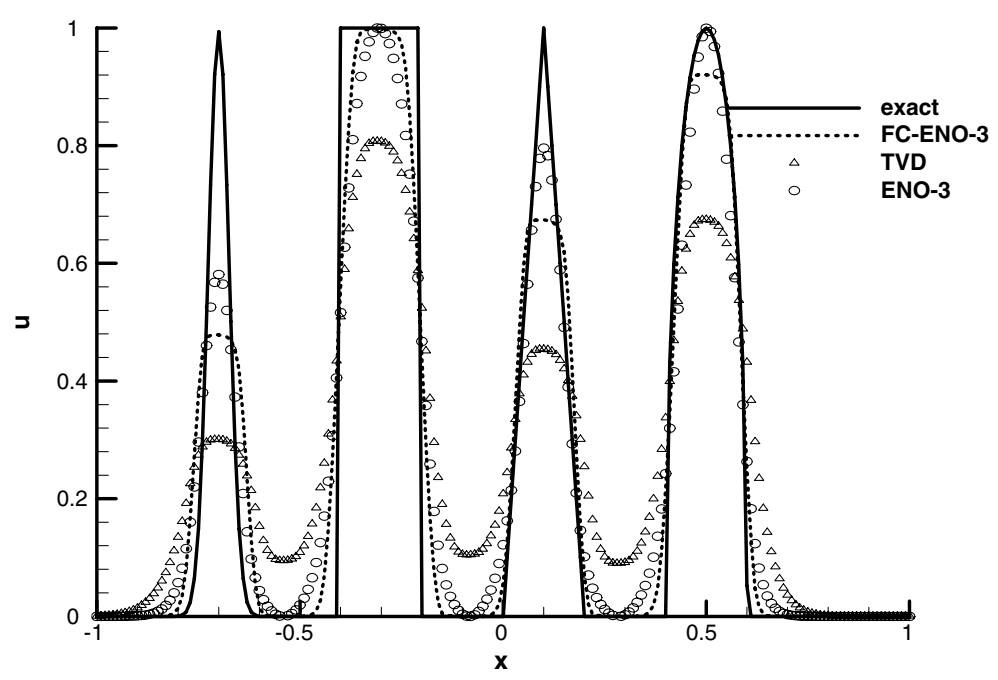

Fig. 5a. Numerical results of example (3), $N=200, t=4$. 


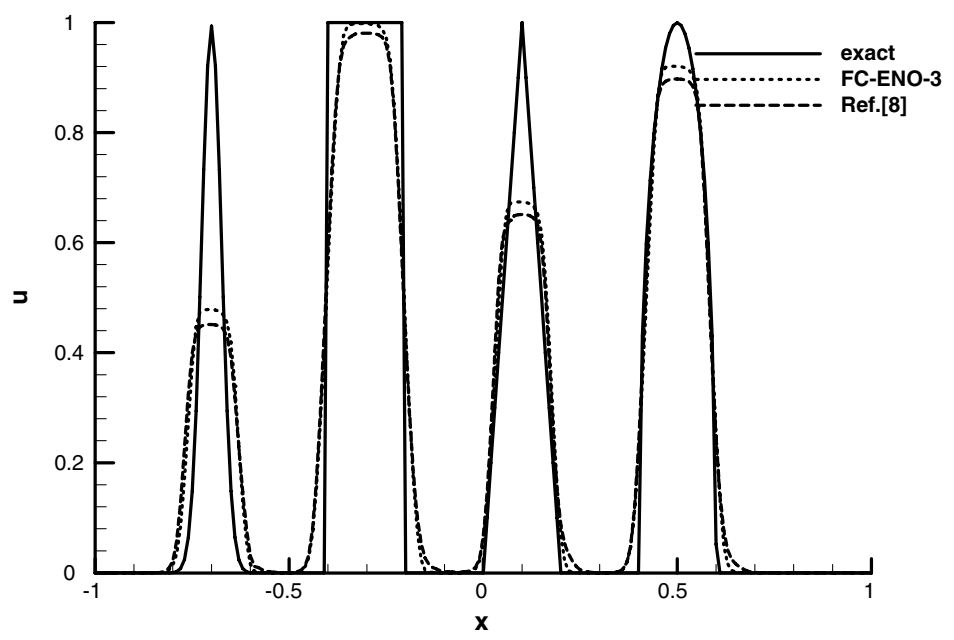

Fig. 5b. Comparisons of FC-ENO-3 and Ravichandran's scheme (Ref. [8]).

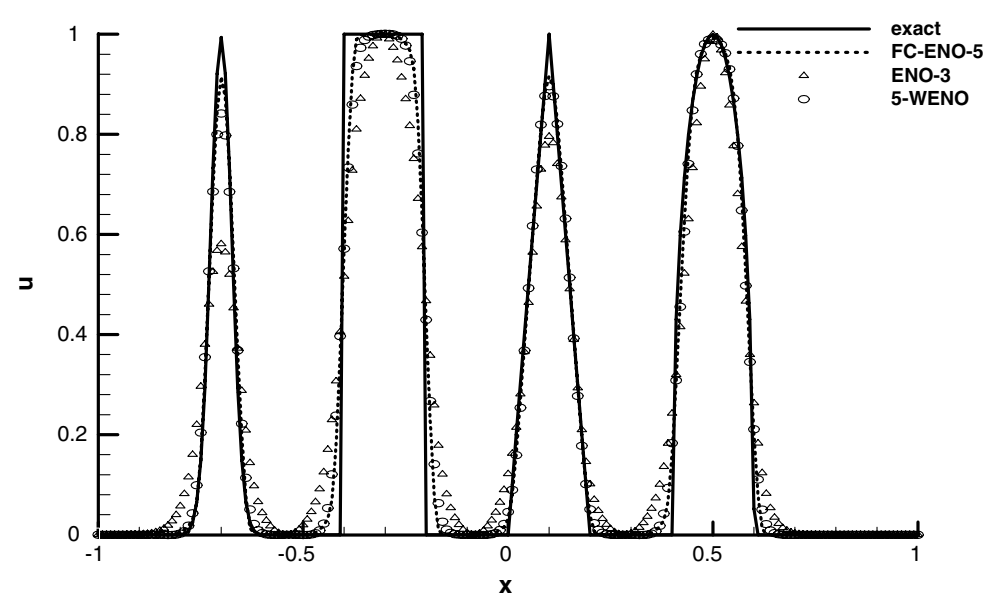

Fig. 5c. Numerical results of example (3), $N=200, t=4$.

(4) Nonlinear transport equation. The nonlinear transport equation is used as the fourth test case and can be written as

$\frac{\partial u}{\partial t}+u \frac{\partial u}{\partial x}=0, \quad 0 \leqslant x \leqslant 2 \pi$

$u(x, 0)=0.3+0.7 \sin x, \quad 0 \leqslant x \leqslant 2 \pi$, with a periodic boundary condition.

The Lax-Friedrichs splitting method is used, in which $f^{ \pm}(u)=\frac{1}{2}(f(u) \pm a u)$ and $a=\max _{u}|f(u)|$. Figs. $6 \mathrm{a}-6 \mathrm{~d}$ show the results at $t=2$ with grid number of $N=80$. For this case, the capability capturing discontinuity of FC-ENO-3 is between TVD and ENO-3 in which more grid points are used (see Figs. $6 \mathrm{a}$ and $6 \mathrm{~b}$ ). The result of FC-ENO-5 is between ENO-3 and 5-WENO, as seen in Figs. 6c and 6d.

In order to check the influence of the high resolution fluxes used in Eq. (13) on accuracy in the case with a discontinuity, we show point-wise errors for grid points of $N=10,20,40,80$ and 160 in Figs. 6e, $6 f$ and $6 \mathrm{~g}$. We can see that these schemes are of a reduced accuracy only in a local region around the shock. Following Eqs. (14) and (20) where there are $h_{j-1 / 2}^{ \pm}=\hat{h}_{j-1 / 2}^{ \pm}+\mathrm{O}\left(\Delta x^{n}\right)$, for the third-order FC-schemes, $n \geqslant 1$; and for FC-ENO-5, $n \geqslant 2$. In Eq. (13), the limited (high resolution) fluxes $h_{j-1 / 2}^{ \pm}$are used at 


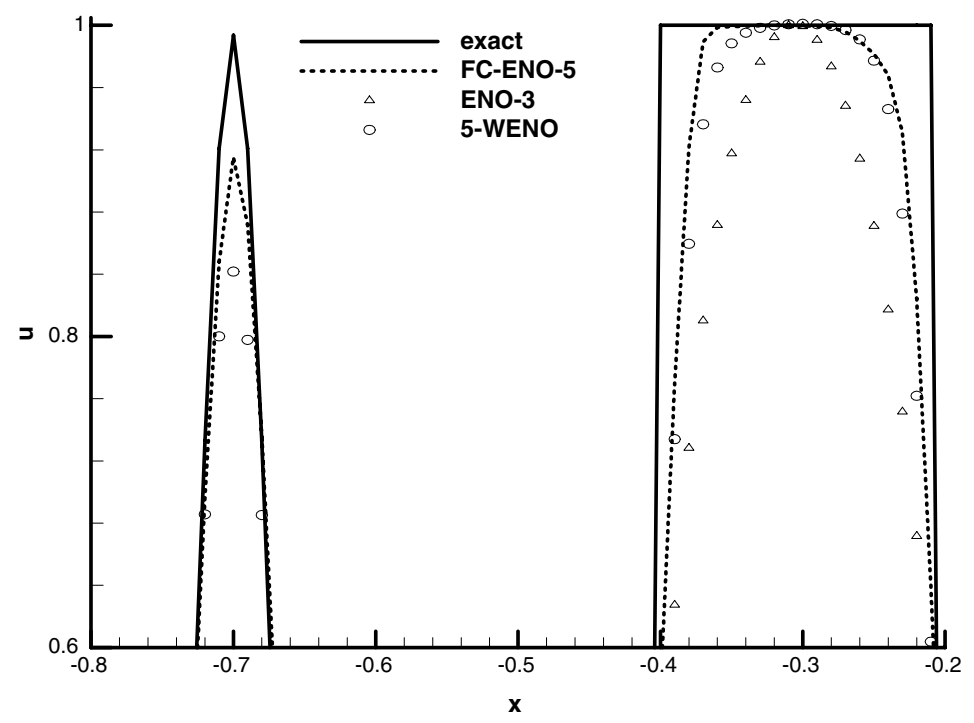

Fig. 5d. The local enlarged part of Fig. 5c.

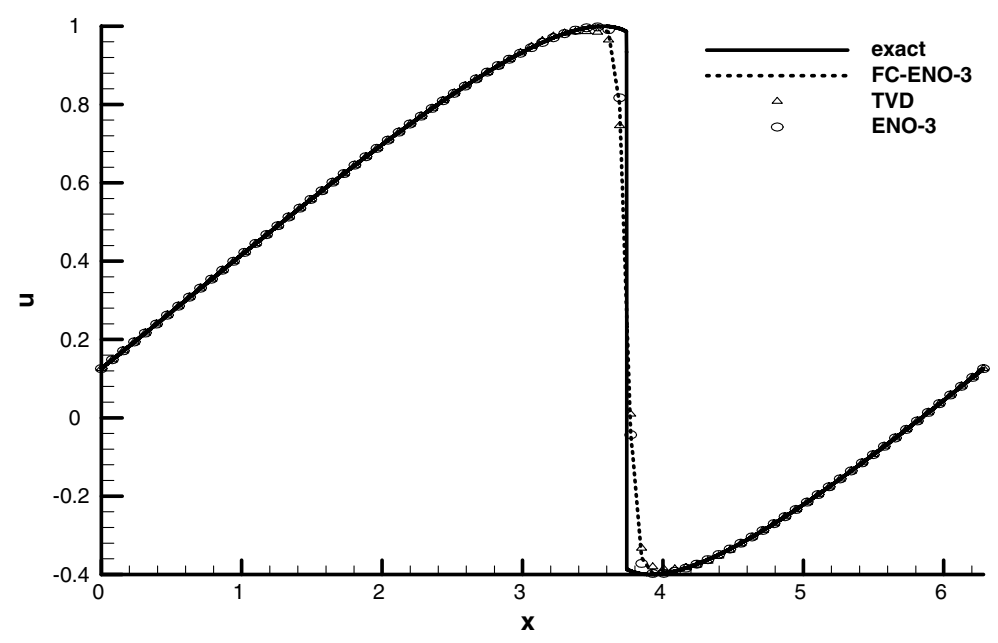

Fig. 6a. Numerical results of example (4), $N=80, t=2$.

the points in a discontinuity, and the coefficients of $h_{j-1 / 2}^{ \pm}$are less than one, i.e., $\alpha^{ \pm} / \beta^{ \pm}<1$. So, in the region away from the discontinuity, the effect of the limited fluxes on the accuracy become smaller and smaller and Eq. (13) can retain its higher order of accuracy.

(5) Viscous Burgers equation. The viscous Burgers equation is our fifth case and written as

$\frac{\partial u}{\partial t}+u \frac{\partial u}{\partial x}=\frac{1}{R e} \frac{\partial^{2} u}{\partial x^{2}}, \quad a \leqslant x \leqslant b$,

$\left\{\begin{array}{l}u(a)=\tanh (-a \operatorname{Re} / 2), \\ u(b)=\tanh (-b \operatorname{Re} / 2) .\end{array}\right.$

The steady solution of Eq. (26a) with boundary condition (26b) is $u(x)=\tanh (-x \operatorname{Re} / 2)$. At $x=0$, the shock is formed with a large Re number. The goal of this test case is to examine the stability and accuracy of the boundary formulas at the presence of a shock at the computational domain boundary. Doing 


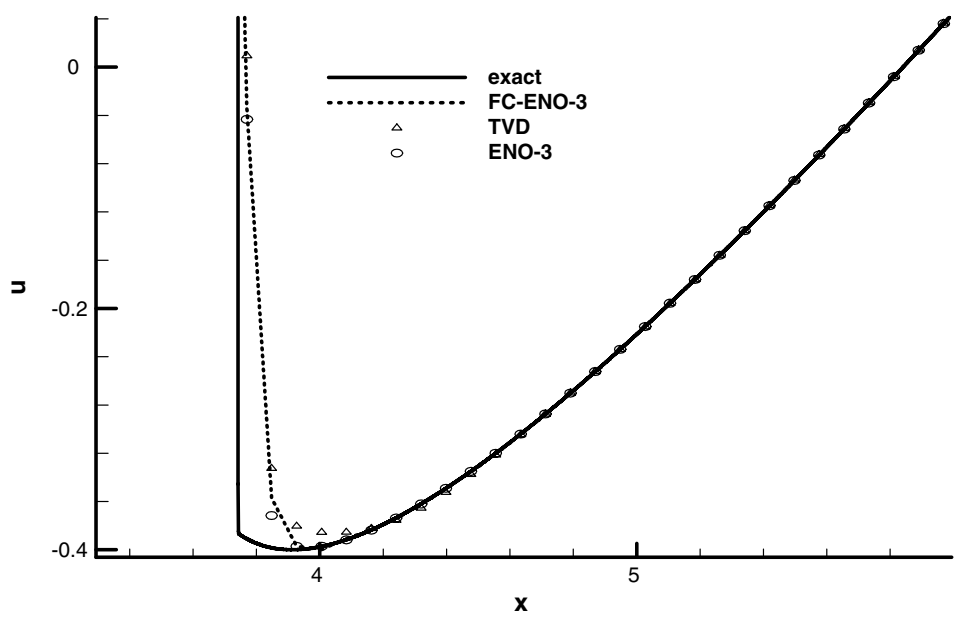

Fig. 6b. The local enlarged part of Fig. 6a.

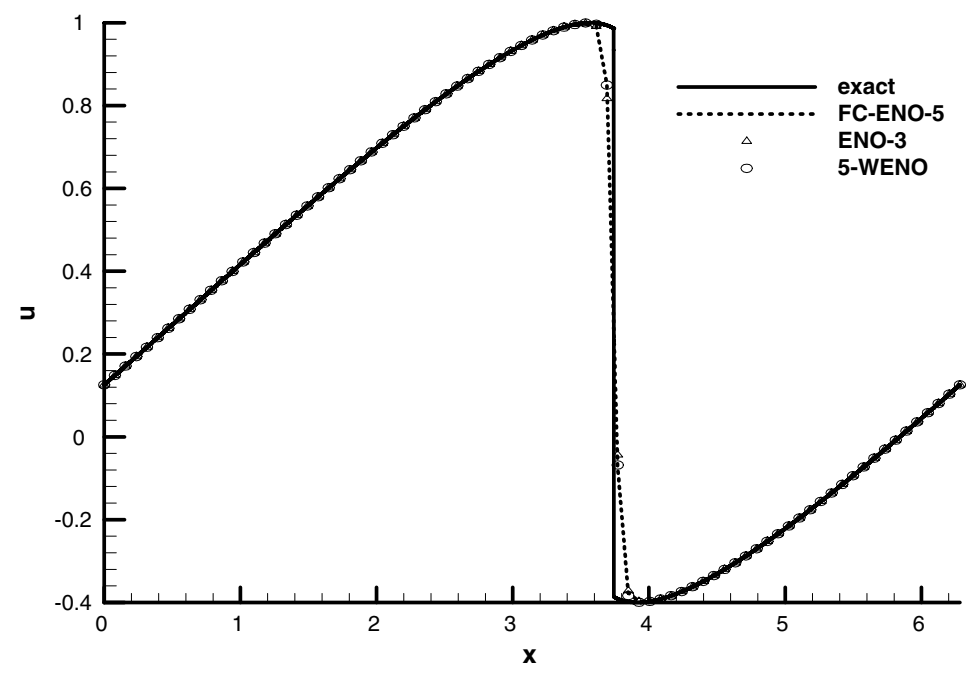

Fig. 6c. Numerical results of example (4), $N=80, t=2$.

so in our computation, we take $a=-1$ and $b=0$, and the grid point number on $N=80$. The central difference scheme is applied for the second-order derivative. The results with $R e=10^{2}$ and $R e=10^{3}$ are shown in Figs. $7 \mathrm{a}$ and $7 \mathrm{~b}$. It can be seen that the boundary formulas are efficient in the case with the shock.

(6) Sod problem. Sod problem is chosen as the sixth test case and its governing equation is one-dimensional Euler equations:

$\frac{\partial \vec{U}}{\partial t}+\frac{\partial \vec{F}}{\partial x}=0$

where $\vec{U}=(\rho, \rho u, \rho e)^{\mathrm{T}}, \vec{F}=\left(\rho u, \rho u^{2}+p, u(\rho e+p)\right)^{\mathrm{T}}, p=(\gamma-1)\left(\rho e-\rho u^{2} / 2\right), \gamma=1.4$. The initial condition is

$(\rho, u, p)= \begin{cases}(1.0,0.0,1.0), & \text { when } x \leqslant 0.5 \\ (0.125,0.0,0.1), & \text { when } x>0.5\end{cases}$ 


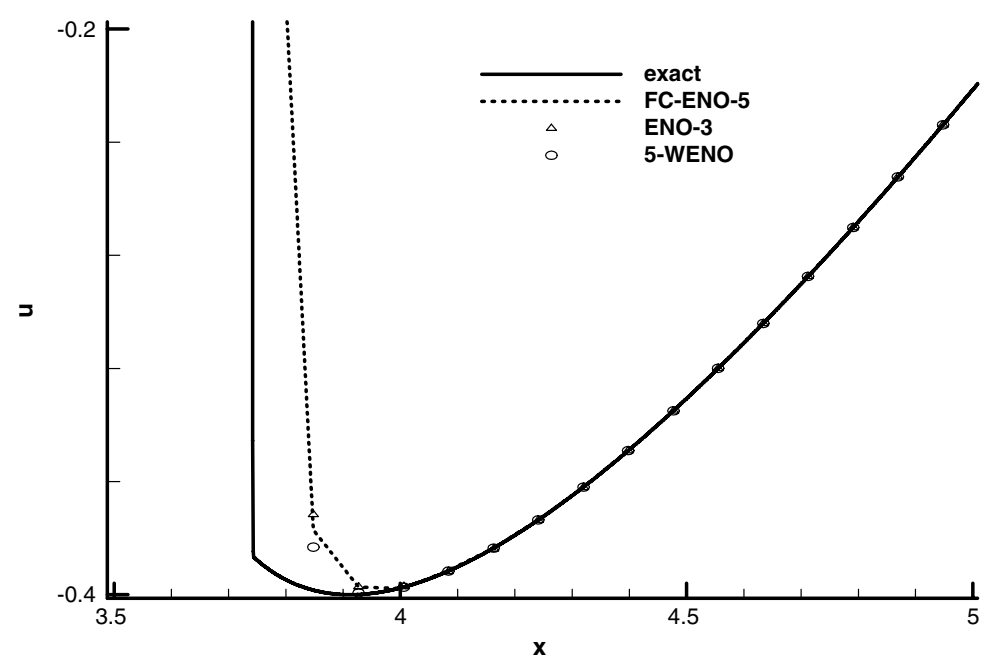

Fig. 6d. The local enlarged part of Fig. 6c.

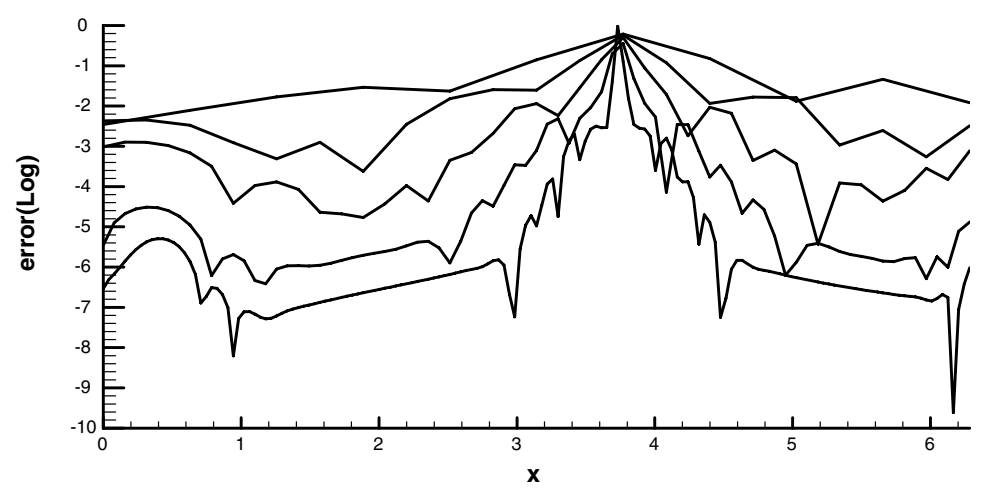

Fig. 6e. Point-wise errors of the FC-TVD scheme.

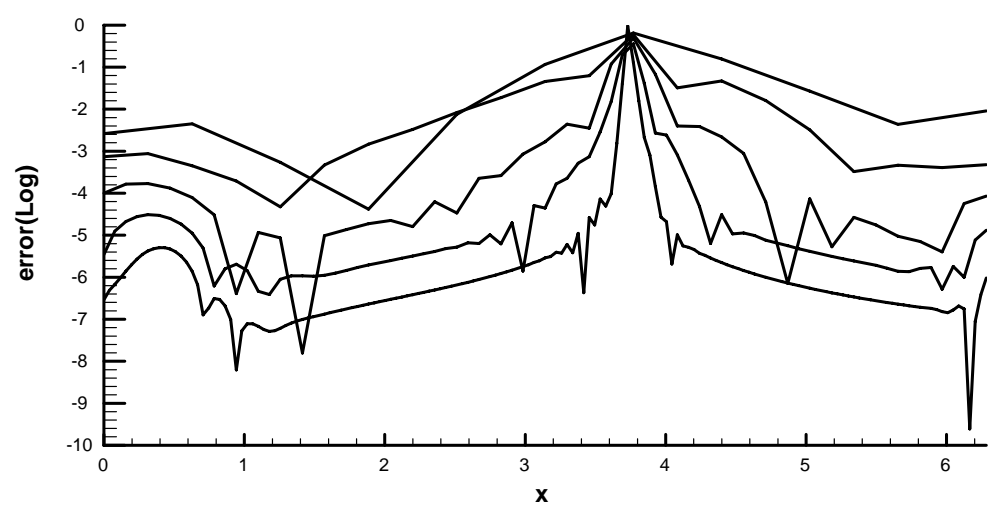

Fig. 6f. Point-wise errors of the FC-ENO-3 scheme.

The initial data are those of the Riemann problem proposed by Sod in Ref. [22], and the case has become a standard test problem for decades, see also Refs. [3-6]. The Steger-Warming flux splitting [21] is used for the equation and results at time $t=0.14$ are shown in Figs. $8 \mathrm{a}-8 \mathrm{~d}$. FC-ENO-3 is better than TVD 


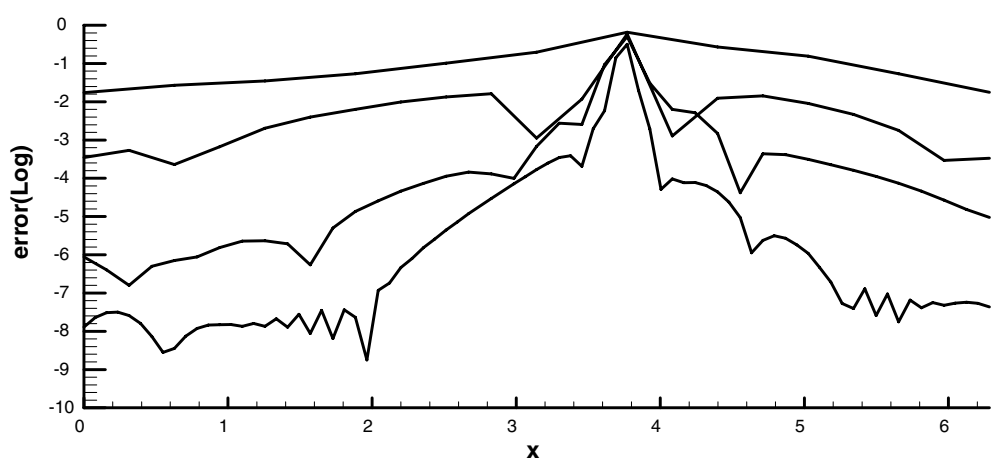

Fig. 6g. Point-wise errors of the FC-ENO-5 scheme.

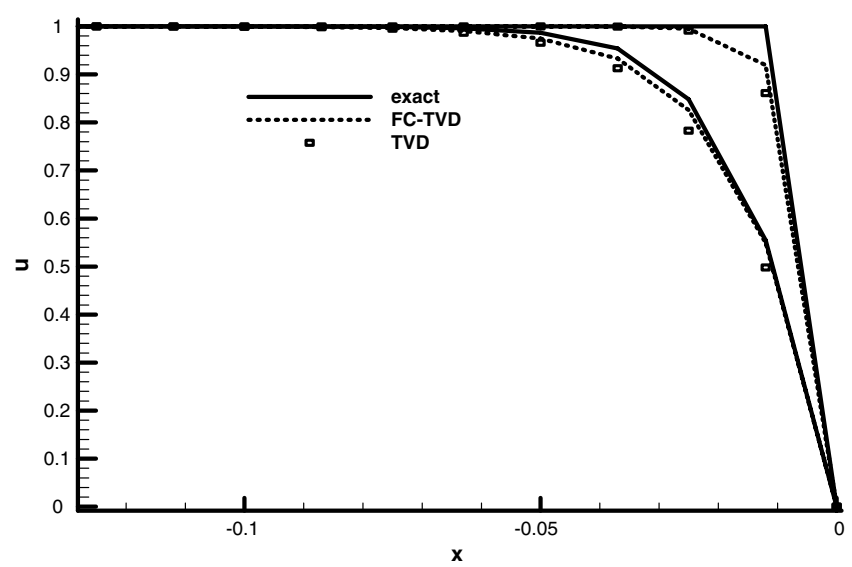

Fig. 7a. Comparison between the FC-TVD scheme and TVD scheme.

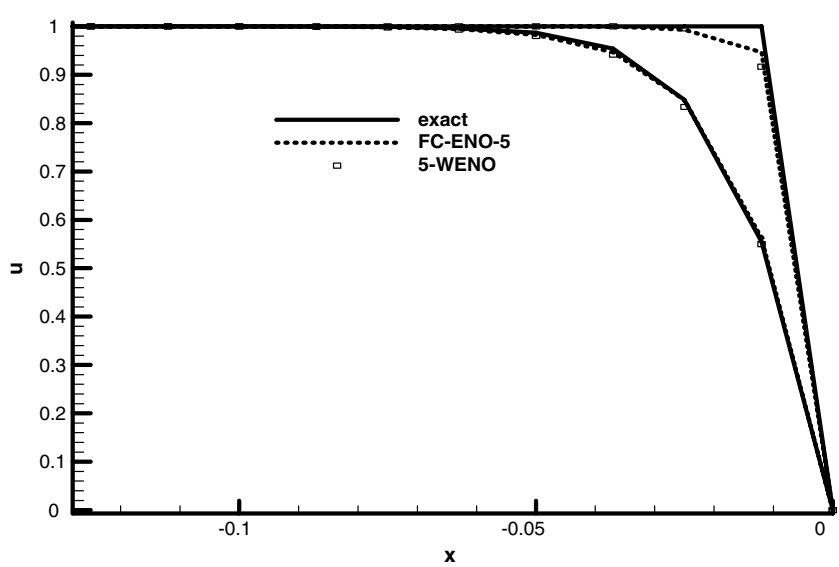

Fig. 7b. Comparison between the FC-ENO-5 scheme and 5-WENO scheme.

and ENO-3, especially for the expansion wave as shown in Fig. 8c. Also, a slightly better improvement of FC-ENO-5 over 5-WENO can be observable in Figs. 8d and 8e.

(7) Shu-Osher problem. Shu-Osher problem is the seventh example and it is governed with one-dimensional Euler equations (27) with following initial condition: 


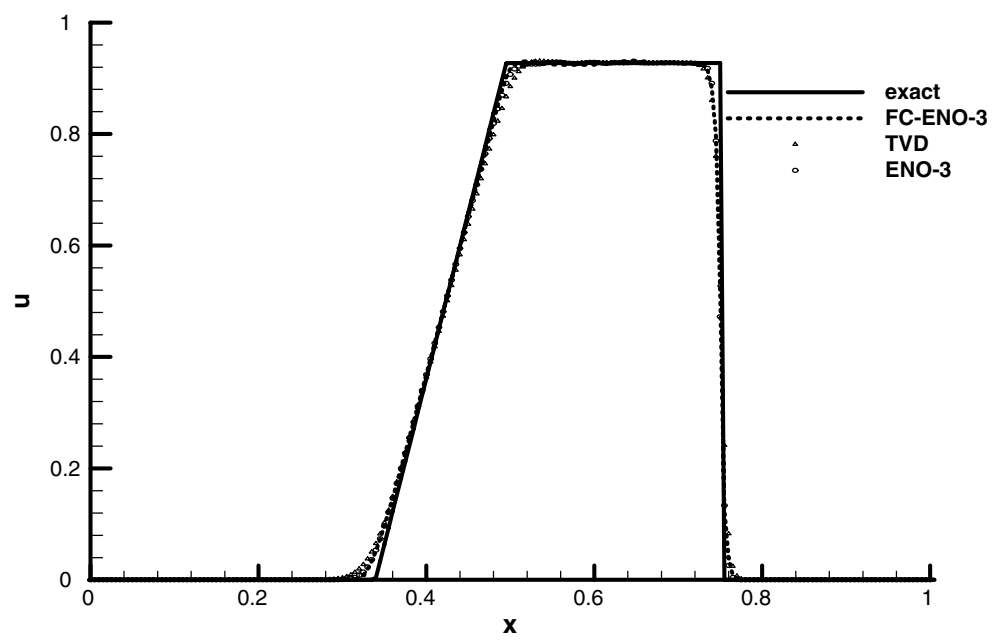

Fig. 8a. Comparisons of velocity of Sod problem.

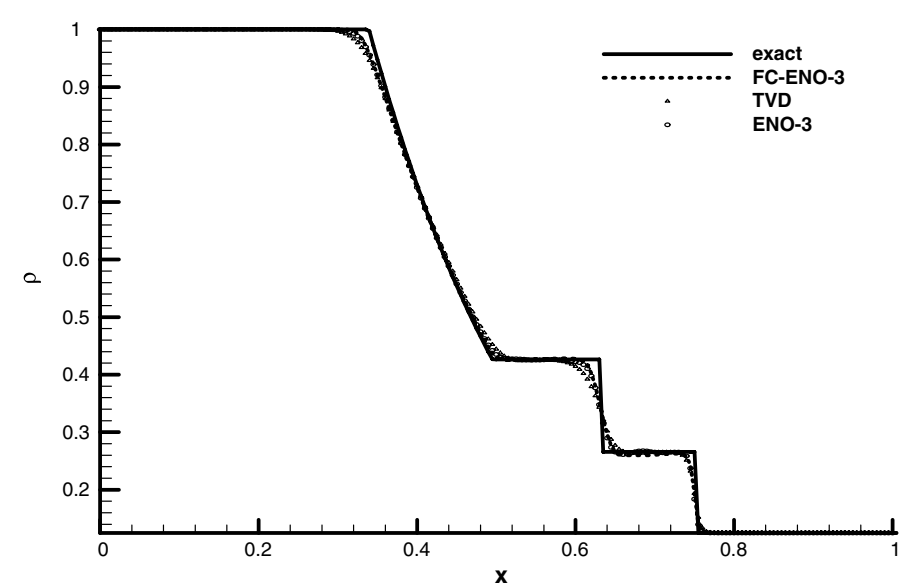

Fig. 8b. Comparisons of density of Sod problem.

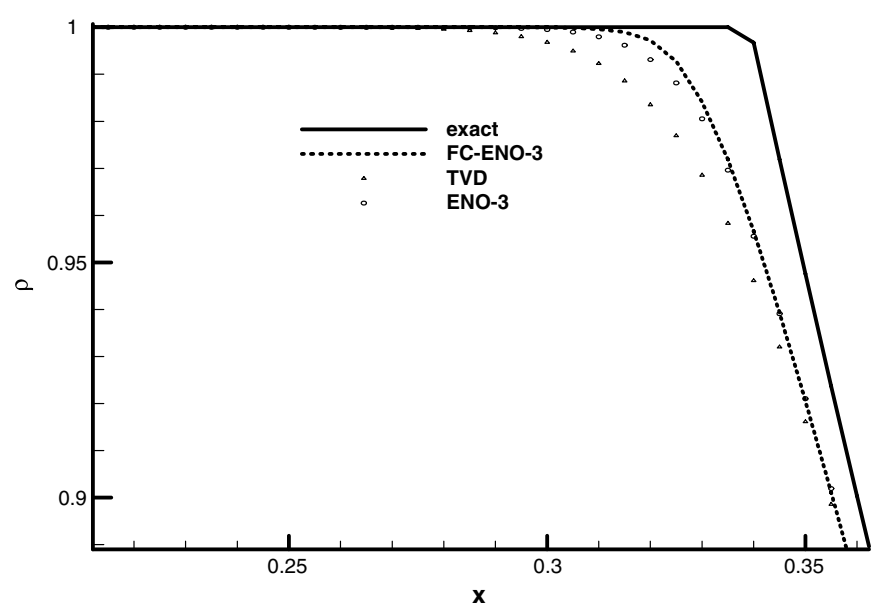

Fig. 8c. The local enlarged part of Fig. 8b. 


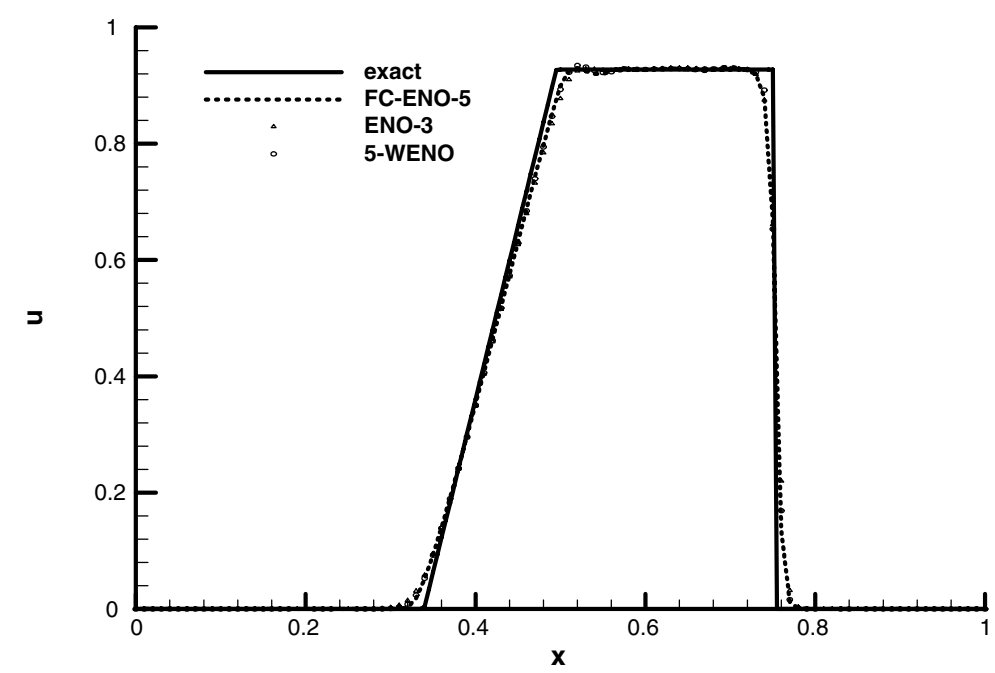

Fig. 8d. Comparisons of velocity of Sod problem.

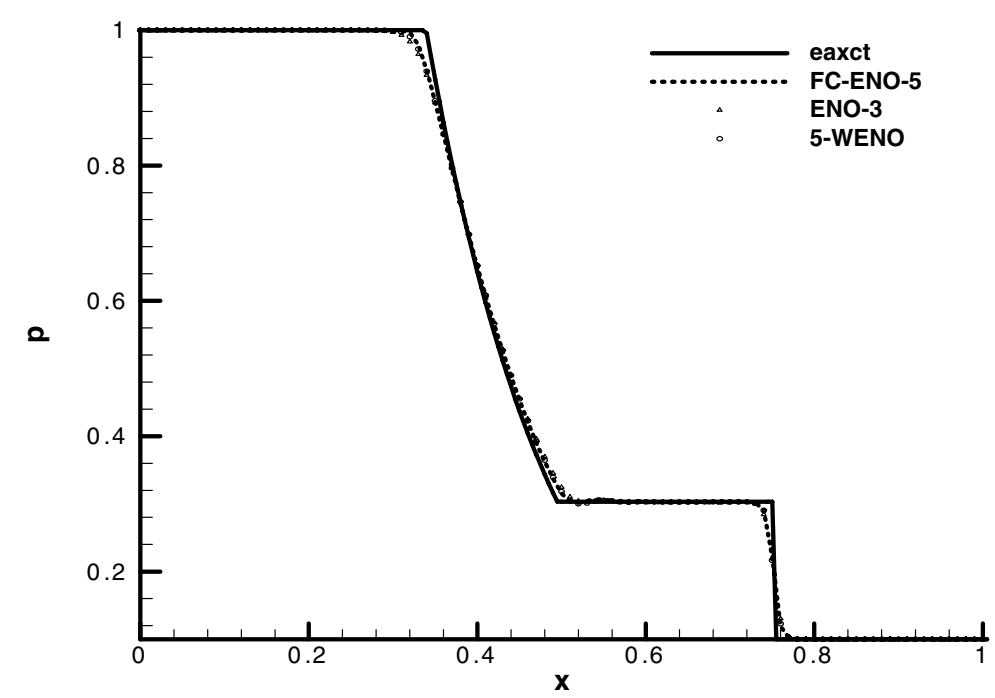

Fig. 8e. Comparisons of pressure of Sod problem.

$$
(\rho, u, p)= \begin{cases}(3.857143,2.629369,10.33333), & \text { when } x<-4, \\ (1+\varepsilon \sin 5 x, 0,1), & \text { when } x \geqslant-4 .\end{cases}
$$

Here, $\varepsilon=0.2$. This test case is taken from Ref. [5], and has been studied by several other authors (see Refs. $[10,11]$ ), and represents a Mach 3 shock wave interacting with a sine entropy wave. The results at time $t=1.8$ are plotted in Fig. 9a-9c. The "exact" solutions (solid lines) are the numerical solutions of WENO-5 scheme with grid points of $N=8000$. For this case, it can be shown that FC-ENO-3 is better than TVD (see Fig. 9b), and FC-ENO-5 is better than 5-WENO (see Fig. 9c).

(8) Two-dimensional linear conservation law with variable coefficients

$$
\frac{\partial u}{\partial t}+\frac{\partial(-y u)}{\partial x}+\frac{\partial(x u)}{\partial y}=0, \quad-1 \leqslant x, y \leqslant 1 .
$$

with periodic boundary conditions. The initial condition is chosen as the characteristic function of a circle with radius 0.5 (Fig. 10a). The problem represents a solid body rotation and is used to investigate 

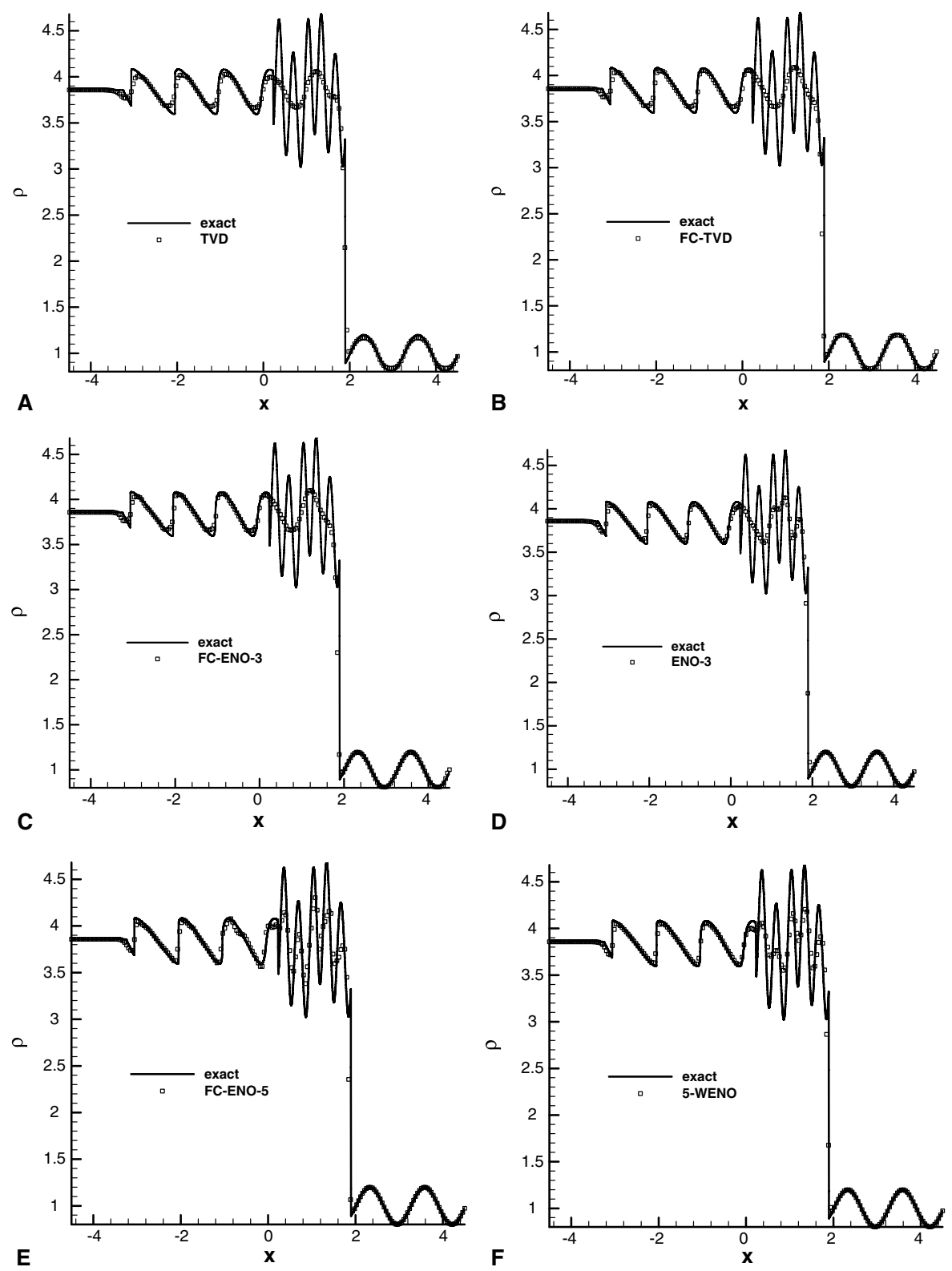

Fig. 9a. Density distributions of Shu-Osher problem with $N=200, t=1.8$. Density distributions calculated by (A) TVD, (B) FC-TVD, (C) FC-ENO-3, (D) ENO-3, (E) FC-ENO-5, (F) 5-WENO.

the grid orientation effect as in Ref. [7]. The results at $t=2$ in a $100 \times 100$ points grid are shown in Figs. $10 \mathrm{~b}$ and $10 \mathrm{c}$. The initial circle stays round after rotation, it indicates that the grid orientation effect is not strong and the result of the FC-ENO-5 is better than FC-ENO-3.

(9) Two-dimensional shock reflection problem. Two-dimensional Euler equations are solved for this problem with following initial condition:

$$
(\rho, u, v, p)= \begin{cases}(1.0,2.9,0.0,0.714286), & \text { ahead of the incident shock, } \\ (1.69997,2.61934,-0.50633,1.52819), & \text { behind the incident shock }\end{cases}
$$

This problem was proposed by Yee et al. [23] and has been widely discussed in the literatures. The incident shock angle is $29^{\circ}$ and a free stream Mach number is 2.9 . The computational domain is [ $\left.4 \times 1\right]$ with uniform 


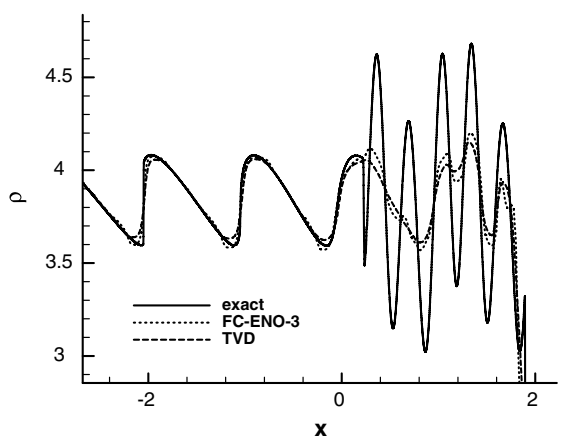

Fig. 9b. Comparison of FC-ENO-3 and TVD, $N=400$ (local enlarged).

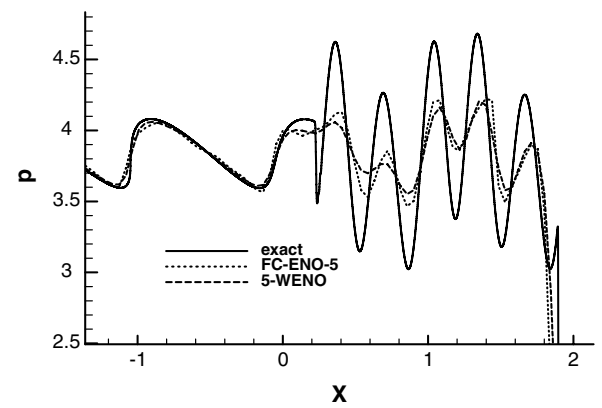

Fig. 9c. Comparison of FC-ENO-5 and 5-WENO, $N=200$ (local enlarged).

grid of $121 \times 41$ points. Steger and Warming's flux vector splitting method [21] are applied to this case. Figs. $11 \mathrm{a}$ and $11 \mathrm{~b}$ show the pressure contours computed by FC-ENO-3 and FC-ENO-5 schemes. From the comparison of the pressure coefficient distribution at $y=0.5$ in Figs. $11 \mathrm{c}$ and $11 \mathrm{~d}$, the FC-ENO-3 is of higher shock resolution than TVD scheme; FC-ENO-5 and 5-WENO give the same result in the region of incident shock; but 5-WENO scheme shows its little downstream position of the reflected shock, and FC-ENO-5 and

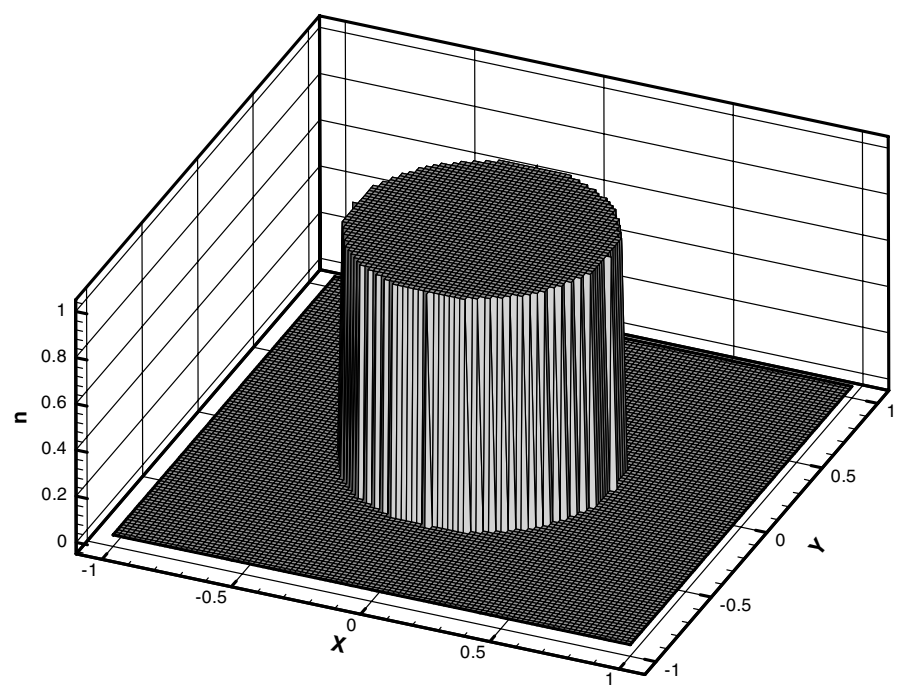

Fig. 10a. Initial condition. 


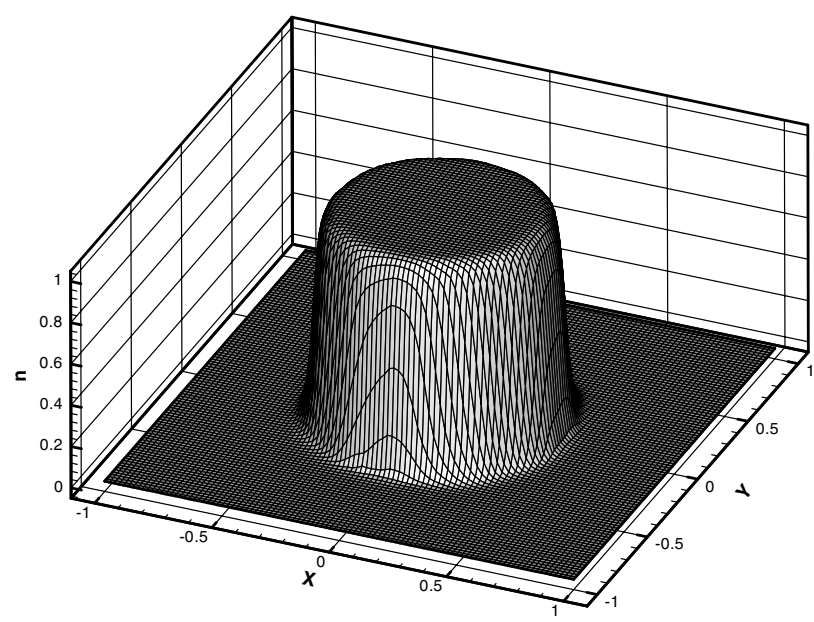

Fig. 10b. Solution of FC-ENO-3, $t=2$.

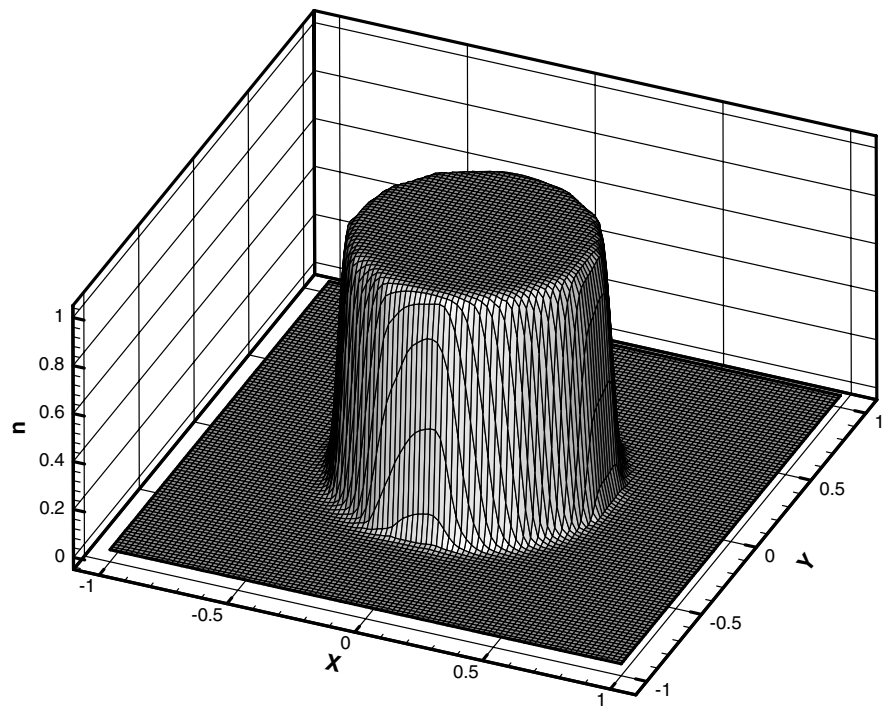

Fig. 10c. Solution of FC-ENO-5, $t=2$.

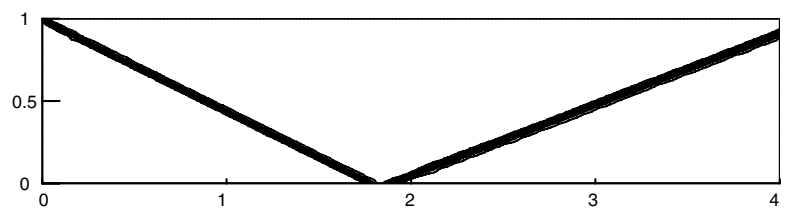

Fig. 11a. Pressure contours, FC-ENO-3 with $121 \times 41$ grids.

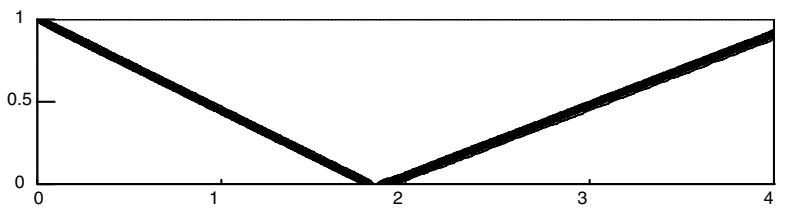

Fig. 11b. Pressure contours, FC-ENO-5 with $121 \times 41$ grids. 


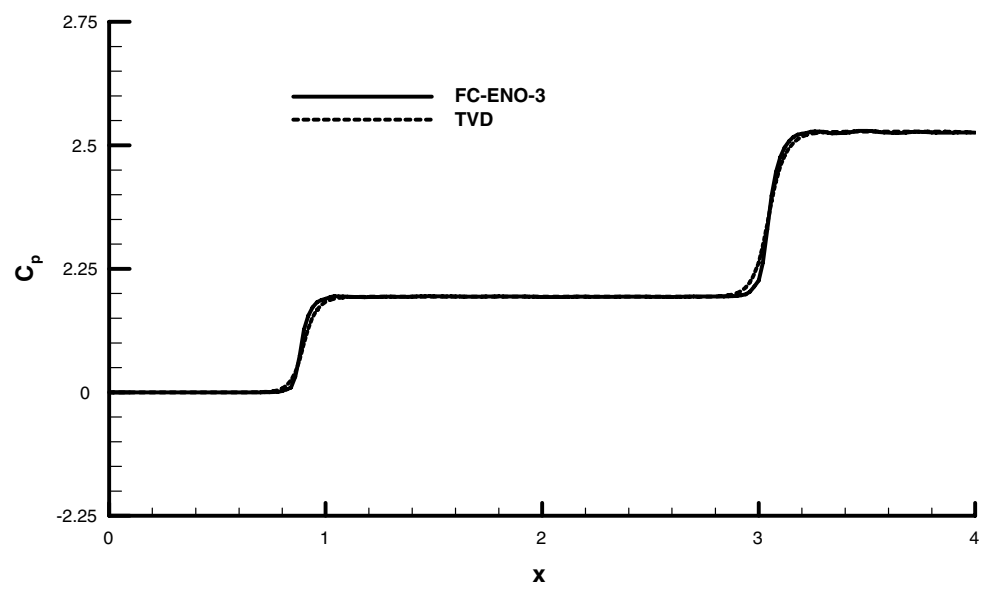

Fig. 11c. Contributions of pressure coefficients (FC-ENO-3 and 2-order TVD) at $y=0.5$.

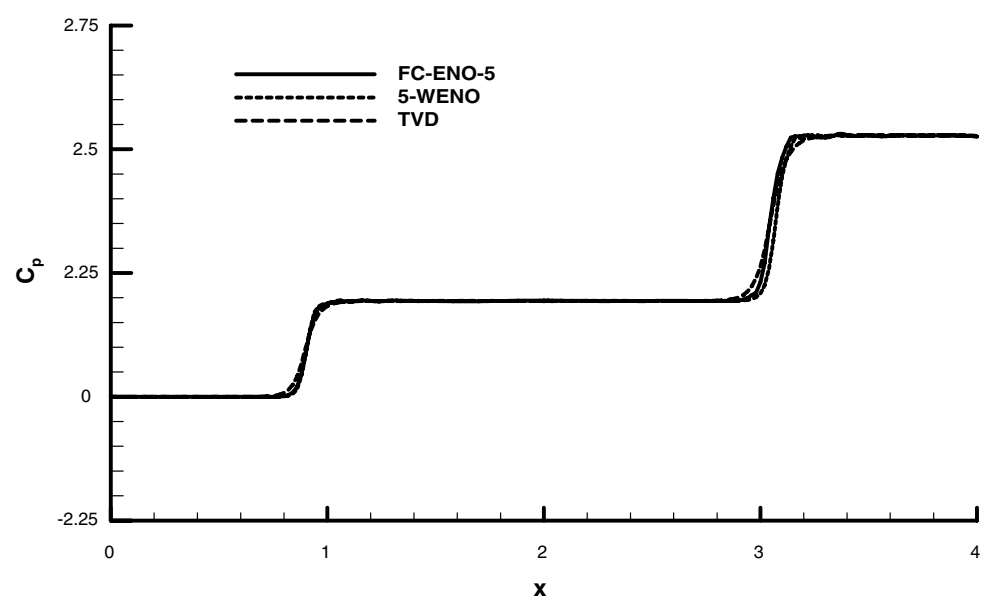

Fig. 11d. Contributions of pressure coefficients (FC-ENO-5, 5-WENO and TVD) at $y=0.5$.

TVD schemes indicate the shock in the same position, however, the resolution of the reflected shock simulated with FC-ENO-5 is evidently better than that with TVD.

\section{Conclusions}

In this paper, a finite compact difference scheme only requiring bi-diagonal matrix inversion is proposed, being much simpler than the commonly used compact schemes with tridiagonal matrix inversion. The known high-resolution fluxes can be used in the compact relations to solve the numerical flux.

By introducing TVD and ENO limiters, the high-resolution and high-order FC-TVD, FC-ENO schemes have been constructed. Numerical results show that the FC-schemes have higher-order of accuracy in smooth region and can avoid spurious numerical oscillations in discontinuous region.

In addition, the boundary formulations, involving one boundary value for FC-TVD and FC-ENO-3, and two boundary values for FC-ENO-5, were suggested. Numerical results demonstrated that the boundary formulations are efficient, and do not influence the accuracy and essentially non-oscillatory property of the FC-schemes. 


\section{Acknowledgment}

This work is supported by the National Natural Science Foundation of China (No. 10402043).

\section{References}

[1] C. Canuto, M.Y. Hussaini, A. Quarteroni, T.A. Zang, Spectral Methods in Fluid Dynamics, Springer, New York, 1987.

[2] S.K. Lele, Compact finite difference schemes with spectral-like resolution, J. Comput. Phys. 103 (1992) 16.

[3] A. Harten, High resolution schemes for hyperbolic conservation laws, J. Comput. Phys. 49 (1983) 357.

[4] A. Harten, B. Engquist, S. Osher, S.R. Chakravarthy, Uniformly high order accuracy essentially non-oscillatory schemes III, J. Comput. Phys. 71 (1987) 231.

[5] C.W. Shu, S. Osher, Efficient implementation of essentially non-oscillatory shock-capturing schemes, II, J. Comput. Phys. 83 (1989) 32.

[6] J.P. Wang, Finite spectral method based on non-periodic Fourier transform, Comput. Fluids 27 (1998) 639.

[7] B. Cockburn, C.W. Shu, Nonlinearly stable compact schemes for shock calculations, SIAM J. Numer. Anal. 31 (3) (1994) 607.

[8] K.S. Ravichandran, Higher order KFVS algorithms using compact upwind difference operators, J. Comput. Phys. 130 (1997) 161.

[9] N.A. Adams, K. Shariff, A high-resolution hybrid compact-ENO scheme for shock-turbulence interaction problems, J. Comput. Phys. 127 (1996) 27.

[10] S. Pirozzoli, Conservative hybrid compact-WENO schemes for shock-turbulence interaction, J. Comput. Phys. 178 (2002) 81.

[11] Y.X. Ren, M. Liu, H.X. Zhang, A characteristic-wise hybrid compact-WENO scheme for solving hyperbolic conservation laws, J. Comput. Phys. 192 (2003) 365.

[12] Z. Jiang, On dispersion-controlled principles for non-oscillatory shock-capturing schemes, Acta Mech. Sin. 20 (1) (2004) 1.

[13] Z. Jiang, K. Takayama, Y.S. Chen, Dispersion conditions for non-oscillatory shock capturing schemes and its applications, Comput. Fluid Dyn. J. 4 (1995) 137.

[14] A.I. Tolstykh, D.A. Shirobokov, Fifth order compact upwind method and its applications to three-dimensional compressible NavierStokes equations, in: Proceedings of the First Asian CFD Conference, Hong Kong, 1995.

[15] Y.W. Ma, D.X. Fu, in: Proceedings of the 5th ISCFD, Sendai, Japan, 1993.

[16] M.H. Carpenter, D. Gottlieb, S. Abarbanel, The stability of numerical boundary treatments for compact high-order finite-difference schemes, J. Comput. Phys. 108 (1993) 272.

[17] A.I. Tolstykh, M.V. Lipavskii, On performance of methods with third- and fifth-order compact upwind differencing, J. Comput. Phys. 140 (1998) 205.

[18] Y.W. Ma, D.X. Fu, T. Kobayashi, N. Taniguchi, Numerical solution of the incompressible Navier-Stokes equations with an upwind compact difference scheme, Int. J. Numer. Meth. Fluids 30 (1999) 509.

[19] G.S. Jiang, C.W. Shu, Efficient implementation of weighted ENO schemes, J. Comput. Phys. 126 (1996) 202.

[20] C.W. Shu, S. Osher, Efficient implementation of essentially non-oscillatory shock-capturing schemes, J. Comput. Phys. 77 (1988) 439.

[21] J.L. Steger, R.F. Warming, Flux vector splitting of the inviscid gasdynamic equations with application to finite-difference, J. Comput. Phys. 40 (1981) 263.

[22] G.A. Sod, A survey of several finite difference methods for systems of non-linear hyperbolic conservation laws, J. Comput. Phys. 27 (1978) 1.

[23] H.C. Yee, R.F. Warming, A. Harten, Implicit total variation diminishing (TVD) schemes for steady-state calculations, J. Comput. Phys. 57 (1985) 327. 